\title{
Chiral Organic Structure-Directing Agents
}

\author{
Luis Gómez-Hortigüela ", Beatriz Bernardo-Maestro
}

Instituto de Catálisis y Petroleoquímica (ICP-CSIC). C/ Marie Curie 2. 28049. Madrid, Spain.Email: lhortiguela@icp.csic.es

\begin{abstract}
Chirality is crucial for life. The preparation of enantiopure chiral compounds is highly desirable in the chemical industry, especially in the pharmaceutical sector. In this context, the design of chiral solids able to discriminate between enantiomers of chiral compounds, either during adsorption or asymmetric catalytic processes, is one of the greatest challenges nowadays in chemical research. Zeolite-type materials represent ideal candidates to achieve enantioselective chiral solids since they could combine their high stability, surface area and shape-selectivity with a potential enantioselectivity that could be enhanced by the confinement effect. Despite the occurrence of chiral zeolite frameworks and the strong interest in preparing these chiral solids, very little success has been met in preparing these in homochiral form. The main strategy to induce chirality in zeolite materials has been the use of chiral structure-directing agents, in an attempt to transfer their chiral feature into the nascent zeolite structure. However, although many chiral organic species have directed the crystallization of zeolite frameworks, some of them even being chiral, there is only one unique very recent example of success in transferring the chirality from the organic structure-directing agent into an enantio-enriched chiral zeolite material. Chiral coordination compounds have been very successful in transferring their chirality onto inorganic frameworks through the development of extensive $\mathrm{H}$-bond host-guest interactions, but these chiral materials usually collapse upon removal of the guest species. In this chapter we report the different types of chiral molecules, both organic and organometallic compounds, used so far as structure-directing agents in an attempt to promote the crystallization of homochiral zeolites; we analyze in detail the possible reasons for the general failure in transferring their chirality, and we propose approaches to prepare known chiral zeolite frameworks in homochiral form. Furthermore, we also review a different approach we have followed in our group in order to induce chirality in zeolite materials, consisting in the development of chiral spatial distributions of dopants embedded in otherwise achiral zeolite frameworks.
\end{abstract}

Keywords chirality, zeolites, templates, structure-directing agents, enantiomer, host-guest chemistry. 


\section{Introduction: Enantiopure chiral zeolitic frameworks, a highly-desired yet elusive target in zeolite science}

Chirality is the property of objects that are not superimposable with their mirror images; the two mirror images are called enantiomers [1]. Depending on the dimensionality where the object is referred, there are several kinds of chiral objects. For instance, a spiral is a chiral object in a 2-dimensional space since it does not superimpose with its mirror image (without leaving the 2-dimensional planar space). The 3-dimensional world where we live is plenty of chiral objects. The classical example is our hands (which indeed gave the name of chirality to this property: the term 'chiral' is derived from 'cheir' meaning 'hand' in Greek), which are not superimposable to each other. Other examples of chirality are those objects containing helicoidal structures, such as propellers, springs or spiral stairs. In nature, chirality is mainly expressed in asymmetric chemical compounds at the molecular level, although asymmetry also manifests sometimes at macroscopic level (see for instance the spiral shells of mollusks). The most frequent expression of chirality is when chemical species have a so-called stereogenic centre, i.e. a tetrahedral atom with four different substituents attached, what gives place (except in particular cases like meso compounds) to species that are not superimposable to their mirror images. In this case, the absolute configuration of the two enantiomers of the chiral compound is determined by the spatial arrangement of those substituents, which gives $(R)$ and $(S)$-enantiomers for each stereogenic centre (following the Cahn-Ingold-Prelog rules [2]). Nevertheless, there are also chiral compounds not based on stereogenic centres but on helicoidal structures, with helicene as the typical example [3].

Chirality is also ubiquitous in living organisms. In fact, homochirality, defined as chirality in its pure enantiomeric form, is crucial for the correct functioning of life. Since its early origin, for some still unclear reason, life in Earth decided to work in an asymmetric fashion [4], although the last origin for this is still a mystery but is a research topic of great interest and current debate [5]. Most of the biochemical building units of living organisms, in particular aminoacids, sugars and nucleotides from which proteins and nucleic acids macromolecules are built, are homochiral [6]. As a consequence, chiral molecules generally trigger a different biological response on living organisms as a function of their absolute stereochemical configuration (i.e. of their enantiomeric form); in fact, very often only one of the enantiomers develops the desired therapeutic effect, while the other enantiomer is less efficient or even no efficient at all [7]. In extreme cases, such as that of the sadly famous thalidomide drug, which was administered to pregnant women in the 1960s, one of the enantiomers $(R)$ had the desired clinical effect, whilst the mirror image $(S)$ triggered teratogenic effects, in that case resulting in malformations in the fetus [8].

Most of the pharmacological drugs administered today are chiral; in 2006, 80 $\%$ of the small-size drugs approved by the U.S. Food and Drug Administration (FDA) were chiral, and $75 \%$ of them were composed of only one of the enantiomers [9]. Hence, one of the greatest challenges today in the chemical industry, es- 
pecially in the pharmaceutical sector, is to obtain and/or separate the pure enantiomeric forms of chiral compounds of interest. In this context, the search for materials able to perform operations in an enantioselective way, i.e. being able to discriminate or distinguish between the enantiomeric forms of chiral molecules, both during asymmetric catalytic operations and in chiral separations, represents nowadays a tremendous challenge of great interest for researchers in applied chemistry. The major benefit of developing this type of enantioselective operations with chiral materials is the chiral multiplication effect, where a minimum amount of a chiral material can give place to large amounts of an enantiomerically-pure (or enantio-enriched) chiral compound of interest [10].

Though much less frequent, chirality is also manifested on inorganic systems. However, very rarely examples of homochirality are found among inorganic compounds: quartz $\left(\mathrm{SiO}_{2}\right)$ was soon recognized to have a chiral structure, however both enantiomorphic crystalline forms of quartz occur in nature in the same (50:50) proportion [11]. In any case, pioneering experiments showed that this type of chiral inorganic systems could transfer their chiral nature into a physicochemical process (separation or catalysis), thus resulting in enantioselective operations, as was discovered by using pure enantiomorphic forms of quartz crystals [12]. Indeed, it has been proposed that minerals with chiral features (like the framework topology or particular crystal surfaces) such as quartz, clays or calcite, could have played a role on the origin of homochirality in the molecules of life $[13,14]$. In this context, zeolite materials (which are nothing else than nanoporous polymorphs of $\mathrm{SiO}_{2}$ ) have been considered as ideal candidates to achieve enantioselective-performing chiral solids since they could potentially combine crucial aspects in chemical processes: their high porosity and large surface area as well as their characteristic shape-selectivity, with enantioselectivity. The shape-selectivity of zeolite materials is provided by their particular porous structure based on channels and/or cavities of molecular dimensions, promoting a confinement effect on guest species adsorbed within the porous systems which greatly influences the outcome of a catalytic reaction. In the same way, such confinement effect could enhance enantioselectivity of a chemical process by reducing the degrees of motion of the guest species, which can only diffuse in particular directions dictated by the porous system of the particular framework structure.

Zeolite materials are typically prepared by hydrothermal methods from concentrated gels in aqueous media, which are then heated in autoclaves at autogenous pressure at temperatures usually ranging between 100 and $200{ }^{\circ} \mathrm{C}$ for a certain period of time. As has been dealt with throughout this Volume, very frequently the synthesis of zeolite materials requires the addition of organic molecules that act as structure-directing agents (SDA) [15]; these molecules organize the tetrahedral inorganic units into a particular geometry from which crystallization of a particular zeolite framework type takes place. In particular occasions, a so-called template effect occurs in which a molecule imprints its size and shape on the nanoporous structure that crystallizes, establishing a clear correspondence between the shape and size of the SDA and that of the porous system. It should be noted that the crystallization process in the presence of these SDA molecules involves that the organic molecules will finally remain occluded within the porous 
systems, and hence they have to be removed in order to empty the pores prior to the use of these materials in adsorption/catalytic processes.

In this context, the shape-selectivity associated to the presence of regular pores and cavities of well-defined dimensions, in a size-scale similar to that of most of chiral organic molecules of interest in the pharmaceutical industry, with the consequent potential to act as molecular sieves, combined with the presence of welldefined isolated active sites, and together with the possibility of modulating the structural properties of the zeolite frameworks through the use of rationallyselected organic SDA molecules, have made zeolite materials one of the most prominent candidates to achieve enantioselective-performing chiral solids. In fact, the search for enantiopure chiral zeolite structures represents one of the greatest challenges in zeolite (and materials) science [16-19], since they could have a great impact on applications such as asymmetric catalysis and enantioselective separations.

The first attempts to induce chirality in zeolite materials consisted on the immobilization of homogeneous chiral catalysts [20] or the anchoring of chiral modifiers [21], which led to notable enantioselective operations. Nonetheless, since the chiral entity is included as an extraframework species in the porous system but is not part of the framework itself, these methodologies often impose problems related to the deactivation or lixiviation of the chiral component during adsorption or catalytic applications. A much more encouraging alternative would be the production of chiral zeolite frameworks where chirality would be intrinsic to the structural network and is not manifested on an extraframework loosely-bound component which would be more weakly retained. In fact, several chiral zeolite frameworks do actually exist [18], like it was long ago recognized with polymorph A of zeolite Beta (BEA) [22], the chiral zincophosphate (CZP) [23], beryllosilicate OSB-1 (OSO) [24], gallogermanate UCSB-7 (BSV) [25], zeolite mineral goosecreekite (GOO) [26], aluminosilicate Linde Type J (LTJ) [27], cobalt-containing aluminophosphate CoAPO-CJ40 (JRY) [28], the recently discovered ITQ-37 (ITV) [29], or SU-32 [30] and HPM-1 [31], germanosilicate and pure-silica forms of the STW framework type. However, despite the occurrence of chiral zeolite frameworks, their main drawback is that they almost invariably crystallize as 50:50 intergrowths of the two enantiomorphic polymorphs (such as polymorph A of zeolite Beta), or as racemic conglomerates, i.e. 50:50 mixtures of both enantiomorphic homochiral crystals, with each individual crystal being enantiomerically pure (such as HPM-1) [32]. Such occurrence of racemic mixtures of the enantiomorphs of chiral zeolite frameworks of course prevents their use in enantioselective operations. Homochirality in the context of chiral inorganic materials very rarely occurs, although some limited but fascinating examples have been reported where homochirality is expressed in a spontaneous, and generally unpredictable, fashion, through symmetry-breaking phenomena [28,33]. On the other hand, the chiral nature of some additional zeolite frameworks has only recently been recognized, and calorimetry results suggest that they could have a potential application in processes of enantiomeric discrimination [34]. It should be remarked here also the work of Zhang and coworkers who developed a very interesting strategy to enrich the 
chiral zincophosphate (CZP) in a particular enantiomer through the use of nucleotides as chiral inductor agents, which will be explained more in detail below [35].

In this line, we cannot continue without mentioning at least the tremendous impact on the topic of chirality in microporous materials triggered by metalorganic frameworks (MOFs). These extraordinary materials are built from metallic centres and organic ligands, giving place to porous coordination polymers with variable pore sizes [36]. These materials have prompted a great revolution in current materials science. The organic nature of the ligands soon promoted the use of chiral ligands to produce chiral MOF materials where the asymmetric entity is part of the framework walls, which indeed led to the production of homochiral solid materials [37,38]; some of them have even led to notable enantioselectivities during adsorption or catalytic processes. In any case, although the development of enantioselective operations with chiral MOF materials is of great interest in current research, these are out of the scope of the present manuscript.

Zeolite networks are built from $\mathrm{TO}_{4}$ tetrahedral units (with $\mathrm{T}$ being typically $\mathrm{Si}$ or $\mathrm{Al}$, although other elements as $\mathrm{P}, \mathrm{Ge}, \mathrm{Ga}$, etc, can also occur); therefore, these units are symmetric, and hence chirality in zeolite materials cannot come from the presence of stereogenic centres as is the typical case for organic chiral compounds. In zeolite materials, chirality arises from the long-range chiral ordering of these $\mathrm{TO}_{4}$ units arranged in chiral space groups, usually in the form of helicoidal channels [18], and not from the building units themselves as in some MOF materials. For this reason, the induction of chirality in zeolite materials has to come from non-framework external units, such as solvents or chiral additives [39]. In this context, the synthesis methodology of zeolites which involves the addition of the organic SDAs provides an obvious strategy in order to induce chirality in these materials through the use of chiral organic species as SDAs. Thus, the main objective here would be to use chiral organic molecules with particular features able to transfer and imprint their asymmetric nature into the long-range chiral ordering of the $\mathrm{TO}_{4}$ units building up a chiral zeolite framework [17].

\section{Chiral organic structure-directing agents: general aspects}

For many years, a large number of chiral molecules has been used as SDAs to direct the crystallization of zeolite frameworks. Many of these have indeed succeeded in directing the formation of zeolite frameworks, some of them even being chiral. Figures 1 and 3 report a list of the organic molecules that have successfully led to zeolite frameworks in silica-based (Figure 1) [17,40-53] and metalophosphate compositions (Figure 3), respectively; Figure 2 shows additional chiral SDAs that were unsuccessful to direct the crystallization of zeolite frameworks.

If a pure enantiomorph of a potential chiral zeolite framework is to be crystallized, of course one needs to use an enantiomerically-pure chiral organic species as SDA in order to promote the transfer of chirality. Typically large amounts of the organic cations are required for the crystallization of zeolite frameworks, which is performed under highly concentrated conditions (especially in fluoride 
media). In this context, enantiomerically-pure chiral compounds are usually very expensive, especially if a chiral separation is required in order to isolate the different enantiomers. Hence, the most obvious strategy to select enantiomerically-pure organic chiral cations as SDAs is to search through the so-called chiral-pool provided by nature. Since living organisms work in an asymmetric fashion, as previously mentioned, enantiomerically-pure chiral natural products are abundant, and usually commercially available at more reasonable costs. In this line, one of the first chiral species used as SDA was derived from $(S)$-sparteine, which is an alkaloid biosynthesized from $L$-lysine that can be extracted from scotch broom [54]. Lobo and coworkers synthesized the N(16)-methyl derivative by quaternization of one of the $\mathrm{N}$ groups (cation $A$ in Figure 1), and observed the crystallization of boron-containing SSZ-24 in sodium hydroxide medium, the silica-analogue of AlPO-5, displaying the AFI framework topology [40]. The authors proposed that the incorporation of boron in the framework was probably related to the hydrophobicity of this cation, which had just one quaternary N. The AFI framework is composed of one-dimensional 12-ring achiral channels, hence evidencing that the chirality of N-methyl-sparteinium was not imprinted on the framework, despite ${ }^{13} \mathrm{C}$ NMR results clearly showing that the cation was incorporated intact within the zeolite structure. Wagner and coworkers used the same cation $(A)$ as SDA, but this time in the presence of lithium hydroxide, and achieved the crystallization of a new zeolite framework, CIT-5 (CFI framework type) [41,55]. CIT-5 contains extra-large 14-ring one-dimensional pores, which together with UTD-1 (DON) were the only high-silica extra-large pore molecular sieves at that time. In line with these works, Tsuji and coworkers also used the N(1)-methyl derivative of another diastereoisomer of spartein, N(1)-methyl- $\alpha$-isosparteinium (cation $B$ in Figure 1), and found that this isomer was more efficient for the crystallization of the same CFI framework in the presence of lithium hydroxide than the previous $(A)$ [43]. With the help of molecular simulations, the authors proposed that the better structure-directing ability of $B$ was because of its ability to adopt a configuration when occluded in the CFI channels that developed more Van der Waals interactions with the zeolite framework than diastereoisomer $A$. However, despite the cation being incorporated intact in the channels, CIT- 1 crystallized in the achiral space group Imma, and hence no transfer of chirality had occurred. In the presence of boron, the AFI framework was crystallized also with this isospartein derivative $(B)$. In the same work, the authors also prepared another sparteine derivative with two quaternary $\mathrm{N}$ ammonium groups $\left(\mathrm{N}(1), \mathrm{N}^{\prime}(16)\right.$-endo-methyl-sparteinium) as SDA, but this dication was unsuccessful to direct the crystallization of zeolite frameworks, probably because of its high hydrophobicity and rigidity. Corma and coworkers have also successfully used the N(16)-methyl-sparteinium (cation $A$ ) for the crystallization of a new zeolite material, ITQ-21, in combination with fluoride anions and germanium in the synthesis gel [42]. ITQ-21 is composed of largecavities and wide pore openings, and exhibited very good catalytic properties. However, again this is not a chiral framework, evidencing once again that the asymmetric nature of the spartein building-block did not play any role during the crystallization process. 
Kubota and coworkers designed a series of chiral SDA cations derived from the natural enantiomerically-pure (-)- $\beta$-pinene monoterpene and its aminocontaining derivative (-)-cis-myrtanylamine [44]. Quaternization of the latter amine through the addition of three methyl groups yielded trimethyl-cismyrtanylammonium (cation $D$ in Figure 1), while trimethyl-transmyrtanylammonium ( $E$ in Figure 1$)$ was prepared by a more elaborate procedure from (-)-trans-myrtanol; both diastereomeric cations were thus prepared in homochiral form. (-)-Cis-myrtanylammonium cation $(D)$ led originally to the production of a new borosilicate zeolite material, CIT-1, with the CON framework type, which was formed by intersecting 10-ring and 12-ring pores [45]; CIT-1 is the ordered polymorph B of SSZ-33. The enantiomer (+)-Cis-myrtanylammonium cation was also prepared and led also to the same CIT-1 material (indistinguishable at least from the XRD patterns), suggesting that the homochirality of the cation did not play a role during the crystallization of the ordered polymorph B in CIT-1; indeed, the space group of the CON framework was achiral (C $12 / \mathrm{m} 1)$. Furthermore, the diastereoisomer trimethyl-trans-myrtanylammonium ( $E$ in Figure 1$)$, as well as the unsaturated derivative trimethyl-myrtenylammonium ( $F$ in Figure 1$)$, also led to the crystallization of the same CON framework in the presence of boron [44]. In line with these SDAs, the authors also prepared another chiral cation with the same myrtanyl polycyclic ring, N,N,N-trimethyltricyclo[5.2.1.0 $\left.0^{2,6}\right]$ decaneammonium (cation $I$ in Figure 1), and in this case they observed the crystallization of SSZ-31 [45], whose structure was later on solved as the interrupted *STO framework type formed as an intergrowth of several polymorphs [51]. Another derivative of (-)-cis-myrtanylamine [(1,1-dimethyl-4-piperidinium)methyl]dimethyl-cis-myrtanylammonium (cation $C$ in Figure 1) directed the crystallization of the MTW framework type, formed by 12-ring one-dimensional channels; however, this cation seems too large to be accommodated within the MTW 1Dchannels, and indeed ${ }^{13} \mathrm{C}$ NMR results showed that in this case the cation did not resist the hydrothermal treatment and fragments produced upon in-situ decomposition of cation $C$ were incorporated within the zeolite [44]. Several other cations derived from the myrtanyl ring were also prepared in the same work (Figure 2) in an attempt to produce zeolite Beta enriched in the chiral polymorph A, but these attempts were unsuccessful and gave no crystalline products under the conditions tested [44].

Kubota and Davis prepared cation $G$ with the enantiopure alkyl substituents (although as a mixture of diastereoisomers as a result of new stereogenic $\mathrm{N}$ centres upon asymmetric quaternization) (Figure 1) [17]. This cation led to the crystallization of all-silica zeolite Beta, but details of this work were not reported. In any case, this report is interesting since it represents one of the few examples published in the literature where zeolite Beta is obtained in the presence of a chiral SDA cation (let us remember that one of the polymorphs of zeolite Beta, A, is chiral, and has not yet been crystallized as a pure polymorph, see section 4.1 below).

Xie and coworkers synthesized cation $H$ through a complex organic synthesis sequence (Figure 1) [46], which was successfully used to prepare the new zeolite SSZ-52. Its framework structure (SFW) was composed of large cavities where pairs of the bulky organic cations were hosted. However, this framework is not 
chiral ( $R-3 m$ space group), and indeed no mention to the asymmetric nature of the organic cation was given in the report.

In an investigation through a high-throughput approach to understand the factors governing the crystallization of extra-large pore zeolites, Jiang and coworkers used cations $J$ and $K$ (Figure 1 ) as SDAs for the synthesis of germanosilicate materials [47]. Both of these cations (OSDAs 3 and 4 in reference 47) contain one stereogenic centre, and hence are chiral compounds. The authors also used two other large SDA cations with stereogenic centres, but these cations had a symmetry plane and hence were achiral meso-compounds (OSDAs 5 and 6). In any case, the authors did not mention about the chirality of those SDAS, and hence it is assumable that they used these chiral compounds as a racemic mixture. Results showed that, depending on the synthesis conditions (amount of $\mathrm{Ge}, \mathrm{Al} / \mathrm{B}, \mathrm{H}_{2} \mathrm{O}$ and OSDA), cations $J$ and $K$ drove the crystallization of zeolite Beta, ITQ-7 and ITQ21. Again the interest here is the crystallization of zeolite Beta, with one of the polymorphs being chiral (A), by using a chiral cation as SDA. Nevertheless, one would not expect any transfer of chirality to the zeolite since racemic compounds were used.

One of the most typical examples of enantiopure chiral natural products derived from the chiral pool is of course provided by $L$-aminoacids. As such, these molecules are not proper SDAs for the synthesis of zeolites because of the presence of the acid group. However, we have been able to produce crystalline AlPObased low dimensional materials by using $L$-lysine as organic guest species because of the presence of an additional amino group in the residue that provides the required basicity [56]; similarly, Dong and coworkers have also produced zinc phosphite/phosphate networks in the presence of $L$-tryptophan and $L$-histidine [57]. If one wants to use these molecules as chiral organic SDAs for the synthesis of zeolite materials, one needs to get rid of such acid group, for instance by reducing the acid to an alcohol group. In our group we selected $L$-proline as chiral precursor because of the rigidity associated to the pyrrolidine ring, which reduces the mobility of the stereogenic centre and hence of the asymmetric group. $L$-proline can be easily reduced to $L$-prolinol by $\mathrm{LiAlH}_{4}$, and the secondary amine can then be alkylated to give an enantiopure quaternary ammonium compound which retains enantiopurity (such alkylation reactions do not involve the stereogenic centre). Moreover, the addition of two different alkyl groups to the $\mathrm{N}$ atom generates a new stereogenic centre with four different substituents attached. Interestingly, depending on the synthesis route employed, we were able to obtain the different diastereoisomers. Starting from $L$-prolinol, the addition of benzyl chloride produces $(S)$-N-benzyl-prolinol; subsequent addition of methyl iodide occurs through only one possible stereochemistry due to the steric constraint provided by the bulky benzyl substituent, giving place exclusively to the $(1 S, 2 S)$-quaternary ammonium cation ((1S,2S)-2-Hydroxymethyl-1-benzyl-1-methylpyrrolidinium) (cation $L$ in Figure 1). In contrast, if the substituent attached first to $L$-prolinol is the small methyl group, subsequent addition of the bulky benzyl group can now take place from both sides (due to the less bulky nature of methyl), hence giving place to a (50:50) mixture of both diastereoisomers [48]. Moreover, we were able to obtain a mixture enriched in isomer $(R, S)$ (up to $77 \%$ ) by successive recrystallizations 
[50]. When the $(S, S)$ diastereoisomer was used as SDA for the synthesis of puresilica zeolites in fluoride media [48], this led to the crystallization of ZSM-12 (MTW); this framework is composed by 12-ring one-dimensional channels. Interestingly, the use of the mixture of isomers $(S, S$ and $R, S)$ as SDA prevented the crystallization of this framework, evidencing that the latter diastereoisomer could not direct the crystallization of the framework, and even inhibited the formation of ZSM-12 by the $(S, S)$ isomer (experiments with the mixture of isomers where the total amount of the $(S, S)$ isomer was the same as in the experiment with enantiopure $(S, S)$ that did lead to MTW also resulted in amorphous materials). Molecular simulations showed a worse fitting of the molecular structure of the $(R, S)$ isomer than that of the $(S, S)$-isomer, thus explaining their different structure-directing efficiency. These diastereomeric compounds also led to the formation of FER-type materials with a certain separation between the FER sheets in the presence of tetramethylammonium as co-SDA [50]. However, the bulky nature of these cations forced the FER sheets to expand a certain distance to accommodate the organic chiral cations between them, a common case in FER-systems, and hence this expanded FER systems could host both diastereomeric cations with no observable differences. In a subsequent work, the $(S, S)$ cation directed also the crystallization of the MWW framework in the presence of tetramethylammonium as co-SDA [49]. All this series of works showed diastereoselective structure-directing effects during the synthesis of zeolite materials, which are expected due to the different shape/size of the diastereoisomers. However, still all the zeolite materials produced were not chiral (MTW, FER and MWW), evidencing once again the difficulty in transferring the chirality from the asymmetric nature of the organic cation into the zeolite framework. In a very recent work, Martínez-Franco et al. have used other quaternary ammonium derivatives of $L$-prolinol (cations $M$ and $N$ in Figure 1), which allowed for the synthesis of Al-containing CDO precursors, but no mention to chirality was given in the report [53].

Finally, very recently Davis and coworkers have successfully used cation $O$ (Figure 1) to promote the enantioselective crystallization of the chiral STW framework [52]; this will be explained in detail in section 4.3 below.

Figure 3 shows the organic chiral species that have been used as SDAs for the synthesis of zeolitic materials based on compositions other than $\mathrm{SiO}_{2}$ [35,58-66]. The synthesis of aluminophosphate-based (AlPO) microporous frameworks is more versatile than that of silica-based materials since it can take place at neutral and acid pHs, hence allowing the use of (usually protonated) amines (rather than quaternary ammonium compounds) which are more efficient SDAs than for the synthesis of zeolites (which usually occurs at higher $\mathrm{pHs}$ where amines remain neutral, though in cases they can also produce zeolites). Following the strategy of searching in the chiral pool, Komura and coworkers prepared the chiral diamine

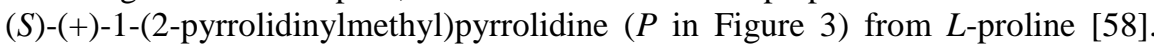
When $P$ was used as SDA for the synthesis of AlPO materials in fluoride medium, a new material denoted GAM-1 was produced. GAM-1 was precursor of the AlPO-CHA framework, and the chiral diamine with two pyrrolidine rings nicely fits within the CHA cages. However, no transfer of chirality from the molecule to the AlPO framework seems to have taken place. 
In the same line, in our group we used (S)-N-benzyl-prolinol ( $Q$ in Figure 3) as an enantiomerically-pure chiral SDA for the synthesis of microporous aluminophosphates [59,60]. A series of MeAPOs materials with the AFI framework was obtained in the presence of different dopants ( $\mathrm{Mg}, \mathrm{Zn}, \mathrm{Co}, \mathrm{Si}, \mathrm{V})$ [59]. This structure is based on one-dimensional 12-ring channels, as previously explained, and hence apparently no transfer of chirality would have occurred. However, we have followed a different approach here in an attempt to induce chirality through chirally-ordered spatial distributions of dopants, which will be explained in section 5 . When we increased the concentration of the organic SDA and the Zn content, we were able to produce the $\mathrm{Zn}$-containing version of the SAO framework for the first time [60]; a similar Zn-SAO material was obtained with the related molecule Nbenzylpyrrolidine, suggesting that the presence of the hydroxymethylene substituent and the stereogenic centre did not play an important role in the crystallization of this achiral framework.

We have also been recently studying the structure-directing efficiency of $(1 R, 2 S)-(-)$-ephedrine and its diastereoisomer $(1 S, 2 S)-(+)$-pseudoephedrine as chiral precursors for the production of AlPO-based frameworks. Ephedrine and pseudoephedrine are alkaloid natural products which are found in nature in the Ephedra species of plants [67], and as such are commercially available in enantiopure form at a reasonable cost. These alkaloids display a biological activity on the sympathetic nervous system, although their pharmacological behavior is different depending on their absolute configuration. We have extensively studied the behavior of these molecules as SDAs for the synthesis of nanoporous aluminophosphates [61,62]. As usual for these phenyl-containing aromatic amines, these molecules led to the crystallization of the AFI framework in the presence of different dopants ( $\mathrm{Mg}, \mathrm{Zn}$ or $\mathrm{Co}$ ); the most stable AFI system was MgAPO-5 [68]. In particular, in order to promote the development of helicoidal arrangements of dopants, as will be explained in section 5 below, we were interested in analyzing the supramolecular behavior of these chiral molecules driven by the development of $\pi-\pi$ type interactions between the aromatic rings on the one side, and of $\mathrm{H}$-bond interactions between the $\mathrm{OH}$ (as $\mathrm{H}$-bond acceptor) and $\mathrm{NH}_{2}{ }^{+}$(as $\mathrm{H}$-bond donor) groups of consecutive dimers packed within the AFI nanochannels. Indeed, a combination of fluorescence spectroscopy and molecular simulations showed that ephedrine displays a much stronger supramolecular trend than its diastereoisomer pseudoephedrine due to a distinct conformational space of the two isomers, which is in turn driven by the development of different intramolecular H-bonds as a consequence of their different stereochemistry [62] (see Figure 4): the most stable conformer of ephedrine involves the alkyl chain in an extended-configuration that enables the supramolecular aggregation, whilst the most stable conformation of pseudoephedrine has the alkyl chain in a folded-conformation that inhibits the formation of dimers (these are only formed under high SDA concentrations). Moreover, such distinct stereochemistry also triggers the occurrence of different orientations of the $\pi-\pi$ stacked dimers of these chiral molecules embedded within the AFI nanochannels [69] (Figure 4). On the other hand, we also added one $((1 R, 2 S)$-methyl-ephedrine) and two $((1 R, 2 S)$-dimethyl-ephedrinium) methyl groups to ephedrine, and observed again the crystallization of the AFI framework 
when using these new chiral derivatives as SDAs [63]; results showed that the hydrothermal resistance of the dimethyl-quaternary ammonium cation was limited. In this case, we observed that the addition of methyl groups involved a notable reduction of the incorporation of supramolecular dimers within the channels. We ascribed this to the more difficult (for methyl-ephedrine) or impeded (for dimethylephedrinium) formation of $\mathrm{H}$-bonds between consecutive dimers when embedded within the AFI frameworks that reduce the repulsive electrostatic interactions established between the closely located positive charges of the $\mathrm{N}$ atoms under the dimer configuration (see Figure 4, bottom-left). In summary, throughout our series of studies analyzing the structure-directing behavior of these chiral derivatives, we have observed interesting packing effects governing the supramolecular chemistry of these molecules during the crystallization of nanoporous frameworks as a consequence of their particular stereochemistry. However, as previously stated, the AFI framework is not chiral itself, which denotes the inability of these molecules (under these conditions) to promote the formation of chiral frameworks. Nonetheless, these molecules are suitable candidates to promote instead the development of chiral spatial distributions of dopants, as will be explained in section 5 .

Although their role has not been that of an organic chiral SDA to direct the crystallization of zeolite frameworks, worth is mentioning the use of two chiral biomolecular additives in the preparation of zeolite materials. The first example is the use of chiral nucleotides as chiral auxiliaries for the enantio-enrichment of crystals of the chiral zincophosphate (CZP) [35]. CZP has an intrinsically chiral topology, which crystallizes as a racemic mixture of crystals (50:50 in $P 6_{1} 22$ or $\mathrm{P6}_{5} 22$ space groups). In their work, the authors proposed the use of a ribonucleotide (uridine-5' -monophosphate, ump, cation $V$ in Figure 3 ) as a chiral additive in order to create homochiral solids or at least enantioenrich the CZP crystals, under the hypothesis that these chiral compounds might develop enantioselective (diastereomeric) interactions with the surface of the CZP enantiomorphic crystals through their phosphate groups. Such interactions of the enantiopure nucleotide should be different with the two enantiomers of CZP, and hence this could potentially lead to a differentiation between the enantiomorphic crystals. Indeed, the authors observed by Circular Dichroism Spectroscopy and single-crystal structural refinements that the addition of a small amount of ump to the crystallization mixture did result in an enantio-enrichment of the CZP crystals in one of the enantiomers (the one in $P 6_{1} 22$ space group, giving an approximate enantiomeric excess of $85 \%$ ). The authors showed the crucial importance of the binding mode of the nucleotide to the growing $\mathrm{ZnPO}_{4}$-based zeotype surface which had to occur through the phosphate group (inosine-5'-monophosphate nucleotide that usually binds to metals through the hypoxanthine ring was not able to promote a similar asymmetric crystallization). This work represented a milestone in the crystallization of enantiopure or enantioenriched chiral zeolite frameworks, although to our knowledge this strategy has not been extended to other systems so far. Nonetheless, in their work Zhang and coworkers have used ump as a chiral auxiliary, but not as a chiral SDA. Of course one rapidly makes the connection between phosphate-containing chiral nucleotides with their potential as SDAs for the synthesis of zeolitic materials, especially those based on $\mathrm{PO}_{4}{ }^{3-}$ networks (such as mi- 
croporous aluminophosphates). These should be ideal candidates to promote the crystallization of chiral microporous AlPO-based frameworks since the asymmetric unit and one of the building units of the zeolitic network $\left(\mathrm{PO}_{4}{ }^{3-}\right)$ are directly linked and an impression of the asymmetric nature of the chiral organic precursor into the zeolite framework should be more easily achievable. Indeed, a direct connection by covalent bonds of $L$-proline aminoacid and phosphate groups has enabled the transfer of chirality from the organic component into a homochiral 3dimensional zinc phosphonate with helical channels [70]. However, the role of these biomolecular nucleotide-based species as SDAs might be severely hindered by the low hydrothermal stability of these compounds (Zhang and coworkers showed that at an intermediate crystallization temperature of $120^{\circ} \mathrm{C}$ the chirality induction effect was lost, probably due to the hydrolysis of the nucleotide into phosphate groups and uridine [35]). In this context, in our group we have used adenosine-monophosphate as SDA for the synthesis of AlPO materials, but these experiments have been unsuccessful so far [71], partly because of its low hydrothermal stability. In any case, diastereomeric interactions between biomolecules essential for life and inorganic crystals have a very strong interest in the scientific community because of their particular relevance to the unsolved problem of the origin of homochirality in the molecules of life [5].

Another important class of chiral biomolecules is provided by $D$-sugars which are part of biopolymers. In this context, there is one chiral sugar derived from $D$ glucose which contains an amino-group that provides the required basicity for SDA molecules, $D$-glucosamine (cation $W$ in Figure 3 ). This molecule has been used for the crystallization of two novel zinc phosphate phases, a mesoporous phase and a lamellar phase [65], but no transfer of chirality was apparent. In another work, we used $D$-glucosamine as additive for the synthesis of SAPO-35 (LEV structure type) in an attempt to promote the formation of mesopores. However, we observed an unexpected behavior: the addition of this amino-containing sugar led to a dramatic reduction of the crystal size [64]. This surprising effect was ascribed to a binding of $D$-glucosamine molecules on the growing surface of the SAPO-35 crystals which hinders further growth, favoring instead the nucleation process and hence the occurrence of small crystals. However, in none of the presented examples the intrinsic chirality of $D$-glucosamine has been transferred to the resulting porous material, but worth is further exploring the ability of these chiral species to transfer their chirality in zeolite frameworks, especially those based on AlPO composition since the crystallization of these is more favourable in the presence of amines (instead of quaternary ammonium cations). In any case, we note again the low hydrothermal stability of $D$-glucosamine under the crystallization conditions that will force the use of low crystallization temperatures, thus reducing the chances of getting 3D-zeolite frameworks.

Although it did not lead to the production of zeolite-type frameworks, for the sake of completeness we also include the use of enantiopure $(1 R, 2 R)-(-)-1,2-$ diaminocyclohexane (cation $X$ in Figure 3) as organic SDA [66]. In this case, the organic chiral amine led to the production of a new low-dimensional gallium phosphate material composed of infinite chains of $\mathrm{GaO}_{6}$ octahedra bridged by phosphate groups, which make the material unstable upon calcination. Interesting- 
ly, when the racemic amine is used, another material is obtained, with the same inorganic chains but a different arrangement of the organic ammonium cations.

In summary, we have presented in this section a list of chiral organic species that have been used as SDAs for the crystallization of zeolite frameworks. Except for one case that will be explained below [52], all these chiral SDAs share the fact that they have not been able to imprint their asymmetric nature onto the nascent zeolite frameworks during the structure-directing phenomenon.

\section{Chiral organometallic structure-directing agents: transfer of chirality from transition-metal complexes through $\mathbf{H}$-bonds}

Another important class of chiral compounds is provided by organometallic compounds, i.e. coordination complexes with transition metals. In these complexes, the ligands are organized around the transition metal in a particular spatial configuration. Their unique chemical properties involve that they can adopt particular conformations that cannot be obtained with usual organic molecules. Interestingly, their geometrical configuration brings the possibility of having chiral coordination complexes. In this case, chirality is not given by the presence of stereogenic centres, as typically in organic molecules, but by the spatial configuration of the ligands. Certain octahedral complexes with a six coordination number in which there are several bi- (or tri)-dentate ligands possess 3-dimensional structures which are not superimposable with their mirror images. Chirality in these compounds is manifested through the spatial configuration whereby the bidentate ligands bind to a given transition-metal in an octahedral environment (see Figure 5). This type of chirality if often referred to as propeller chirality: a right-handed twist of a propeller is not superimposable to its left-handed version (Figure 5-bottom).

This type of organometallic compounds has been extensively used for the synthesis of zeolitic materials usually based on compositions other than silica (mainly aluminophosphates and gallogermanates) [72-81]. Of course for these metal complexes to act as SDA species, they must be stable under the hydrothermal synthesis conditions, what precludes the use of a large number of complexes where an exchange of ligands would readily occur. Figure 6 reports the most commonly used chiral octahedral complexes, which are stable enough to resist the hydrothermal synthesis conditions.

Balkus et al introduced the use of metallophthalocyanines coordination complexes to produce zeolites with the FAU structure, although not all the cavities were occupied with these complexes [82]. Since then, many studies have analyzed the use of chiral coordination complexes as chiral SDAs. The essential requirement for these complexes is that they must resist the crystallization process without racemizing. In this regard, several chiral octahedral complexes with $\mathrm{Co}^{3+}$ cations are kinetically stable under hydrothermal conditions, and hence have been successfully used for the preparation of low-dimensional aluminophosphate, gallophosphate and zincophosphate-based materials (see Figure 6). Morgan and 
coworkers used the $\mathrm{Co}(\mathrm{III})$ tris(ethylendiamine) $\left[\mathrm{Co}(\mathrm{en}){ }_{3}{ }^{3+}\right]$ complex (cation $A$ in Figure 6) as SDA and produced a low-dimensional chiral AlPO framework $\left[\mathrm{Co}(\mathrm{en})_{3} \mathrm{Al}_{3} \mathrm{P}_{4} \mathrm{O}_{16} \cdot 3 \mathrm{H}_{2} \mathrm{O}\right.$ ] [72], in which the chirality of the organometallic complex was directly imprinted onto the aluminophosphate layers. In this type of lowdimensional AlPO frameworks, $\mathrm{Al}$ is usually linked to $4 \mathrm{P}$ atoms (through $\mathrm{O}$ bridges), whilst $\mathrm{P}$ is usually bonded to less than $4 \mathrm{Al}$ (through $\mathrm{O}$ bridges), giving place to terminal $\mathrm{PO}^{-}$groups. In this material, the $\mathrm{Al}_{3} \mathrm{P}_{4} \mathrm{O}_{16}$ framework formed a unique macroanionic sheet, and the chiral $\mathrm{Co}(\mathrm{en})_{3}{ }^{3+}$ cations and water molecules were located in the space between the layers. In the $\mathrm{Al}_{3} \mathrm{P}_{4} \mathrm{O}_{16}$ sheets, the 4-rings had a tricyclic structural motif which resembles a [3.3.3] propellane, hence providing a chiral feature (chiral pockets) where the chiral $\mathrm{Co}(\mathrm{en})_{3}{ }^{3+}$ cations sit. However, the use of racemic $D L-\mathrm{Co}(\mathrm{en})_{3}{ }^{3+}$ involved that both enantiomers were included in the material, and hence overall the crystal structure of this layered AlPO framework was not chiral: it contained both enantiomers of the complex in the same amount, and hence the same number of chiral pockets of opposite handedness. Nonetheless, the discovery of this framework provided evidence that chiral organometallic complexes were able to induce chiral features in an inorganic lattice. In fact, the authors found that such a transfer of chirality must be directly related to the extensive H-bond network established between the complex (through the $\mathrm{NH}_{2}$ groups) and the terminal $\mathrm{PO}^{-}$groups. Bruce and coworkers used in the same time $\mathrm{Co}(\mathrm{III})$ tris-(1,3diaminopropane) $\left[\mathrm{Co}(\mathrm{tn}){ }_{3}{ }^{3+}\right]$ (cation $B$ in Figure 6) to produce a new $\mathrm{Co}(\mathrm{tn})_{3} \cdot \mathrm{Al}_{3} \mathrm{P}_{4} \mathrm{O}_{16} \cdot 2 \mathrm{H}_{2} \mathrm{O}$ low-dimensional framework [73], where again the $\mathrm{Co}(\mathrm{tn})_{3}{ }^{3+}$ cations were located in the interlayer space between the AlPO anionic layers. Remarkably, in this new material each single crystal contained only one enantiomer of the complex despite the use of a racemic mixture in the synthesis, providing an example of spontaneous resolution to give a racemic conglomerate (a mixture of crystals each containing a single enantiomer). This material was a chiral solid, its chirality being due to the presence of such single enantiomer of the complex between the layers, but the chirality was not transferred to the inorganic framework. In a subsequent work, Bruce and coworkers used trans-Co-bis-(2-aminoethyl)amine) $\left[\mathrm{Co}(\mathrm{dien}){ }_{2}{ }^{3+}\right]$ complex (cation $C$ in Figure 6) to produce a new chiral low dimensional material, $\mathrm{Co}$ (dien) $)_{2} \cdot \mathrm{Al}_{3} \mathrm{P}_{4} \mathrm{O}_{16} \cdot 3 \mathrm{H}_{2} \mathrm{O}$ [74] (space group $P 6_{5} 22$ ). This material consisted of chiral aluminophosphate macroanionic layers stacked in a helical fashion occluding a single enantiomer of $\left[\mathrm{Co}(\mathrm{dien})_{2}{ }^{3+}\right]$ between the layers (see Figure 7-top), although again the use of a racemic mixture involved the production of a crystalline racemic conglomerate (a 50:50 mixture of homochiral crystals with opposite handedness). Nonetheless, the incorporation of a single enantiomer of the complex on each crystal involved a strong chiral molecular recognition phenomenon during the crystal growth. Again H-bond interactions (see dashed circles in Figure 7) were believed to play a fundamental role during the transfer of chirality from the complex to the chiral layer.

In a step further, Gray and coworkers produced a new chiral aluminophosphate framework based on a stacking of chiral macroanionic $\mathrm{Al}_{3} \mathrm{P}_{4} \mathrm{O}_{16}{ }^{3-}$ sheets similar to those found previously, but in this case they used enantiomerically-pure $d$ $\mathrm{Co}(\mathrm{en}) 3_{3}{ }^{3+}$ as the SDA (cation $A$ in Figure 6) [75]. The main interest of this new 
material was that there was only one enantiomer of the chiral AlPO sheet in any crystal of the material, with only enantiomer $d$ - $\mathrm{Co}(\mathrm{en}) 3^{3+}$ intercalated between the chiral AlPO sheets (see Figure 7-bottom). Interestingly, the chiral $\mathrm{Al}_{3} \mathrm{P}_{4} \mathrm{O}_{16}{ }^{3-}$ sheets here were different than those of $\left[\mathrm{Co}(\mathrm{en})_{3} \mathrm{Al}_{3} \mathrm{P}_{4} \mathrm{O}_{16} \cdot 3 \mathrm{H}_{2} \mathrm{O}\right.$ ] [72] mentioned previously, prepared with the same cation but in a racemic form (remember that in the latter a mixture of both enantiomers was present in the framework). Instead the AlPO sheets in the present case were similar to those prepared with $\left[\mathrm{Co}(\operatorname{dien})_{2}{ }^{3+}\right]$ (where only one enantiomer was present on each single crystal) [74]. This work provided evidence that homochiral inorganic frameworks can be built using homochiral structure-directing guest species. As usual, an extensive H-bond network seemed to be responsible for the transfer of chirality to the chiral inorganic sheets (see dashed circles in Figure 7-bottom).

Optically pure $d-\operatorname{Co}($ en $) 3^{3+}(A$ in Figure 6$)$ has also led to the production of a chiral 1-dimensional aluminophosphate chain compound, $d$ $\mathrm{Co}(\mathrm{en})_{3}\left[\mathrm{AlP}_{2} \mathrm{O}_{8}\right] \cdot 6.5 \mathrm{H}_{2} \mathrm{O}$ (AlPO-CJ22) [78] (Figure 8). The framework structure of AlPO-CJ22 was similar to that of the previously reported $\mathrm{Co}(\mathrm{en})_{3}\left[\mathrm{AlP}_{2} \mathrm{O}_{8}\right] \cdot \mathrm{xH}_{2} \mathrm{O}[83]$, in which racemic $\mathrm{Co}(\mathrm{en})_{3}$ was disordered between the chains. In contrast, the $d$ - $\mathrm{Co}(\mathrm{en}) 3^{3+}$ cations in AlPO-CJ22 displayed a chiral order in which they follow a $2_{1}$ screw axis along the [100] direction, interacting with the AlPO chains through extensive H-bonds in a helical fashion between the $\mathrm{N}$ atoms and the terminal $\mathrm{O}$ atoms of the inorganic chain (see Figure 8, inset). Indeed, measurement of the optical rotation clearly revealed that the Co complex cations have predominantly the $\Lambda$-configuration. Reversibly, optical rotation measurement of the corresponding material produced with $l$-Co(en $)_{3}{ }^{3+}$ showed the predominance of the Co complexes in the $\Delta$-configuration [78].

Chiral framework materials with other compositions such as gallophosphates [84,85], borophosphates [86] or zincophosphates [76,77,79] have also been prepared in the presence of chiral Co complexes. Racemic $\mathrm{Co}(\mathrm{en})_{3}{ }^{3+}(A$ in Figure 6) led to the discovery of two zincophosphate materials, $\left[\mathrm{Co}^{\mathrm{II}}(\mathrm{en})_{3}\right]_{2}\left[\mathrm{Zn}_{6} \mathrm{P}_{8} \mathrm{O}_{32} \mathrm{H}_{8}\right]$ and $\left[\mathrm{Co}^{\mathrm{III}}(\mathrm{en})_{3}\right]\left[\mathrm{Zn}_{8} \mathrm{P}_{6} \mathrm{O}_{24} \mathrm{Cl}\right] \cdot 2 \mathrm{H}_{2} \mathrm{O}[76]$; both frameworks contained chiral structural motifs, whose chiral features were transferred from the metal complex, thus resulting in a spontaneous separation of the enantiomers of $\mathrm{Co}(\mathrm{en}) 3^{3+}$ as $\Lambda$ - and $\Delta$ configurations in the structure in alternate interlayer regions. In fact, the orderly separation of the enantiomers of the Co complex clearly involved that the inorganic sheets had chiral molecular recognition ability for the asymmetric nature of the Co complex. Such recognition ability was again promoted by the extensive hostguest H-bond network established. Indeed, Wang and coworkers [77] clearly demonstrated that such chiral enantiospecific molecular recognition ability was a consequence of the development of extensive H-bonds between the inorganic chiral motif and the amino-groups of the chiral complexes: such stereospecific correspondence was due to the fact that the number of $\mathrm{H}$-bonds is maximized in a particular diastereomeric interaction (between one enantiomer of the chiral inorganic motif and one enantiomer of the complex), and this number of H-bonds is reduced if the alternative diastereomeric interaction (between one enantiomer of the chiral inorganic motif and the opposite enantiomer of the complex) is established, thus decreasing the stability of the system; this had as a consequence a transfer of chi- 
rality of the complex to the inorganic framework. Interestingly, Wang and coworkers also managed to prepare a new zincophosphate framework using a racemic mixture of $\mathrm{Co}\left(\right.$ dien) ${ }_{2}{ }^{3+}$ (cation $C$ in Figure 6) where the main interest was that it was not a low-dimensional framework material as those mentioned before, but consisted instead of a 3-dimensional open framework $\left(\left[\mathrm{Zn}_{2}\left(\mathrm{HPO}_{4}\right)_{4}\right]\left[\mathrm{Co}(\text { dien })_{2}\right] \cdot \mathrm{H}_{3} \mathrm{O}\right)$ with multidirectional helical 12-ring channels [79] (see Figure 9). In this structure, each 12-ring helical channel accommodated one $\mathrm{Co}(\mathrm{dien}){ }_{2}{ }^{3+}$ cation, indeed a pair of enantiomers in alternating rows. The most attractive structural feature of this open-framework was that the 12-ring channels are enclosed by two intertwined helices of the same handedness (yellow and green polyhedra in Figure 9-right), connected through them by Zn-O-P linkages. Although the presence of both enantiomers of the chiral Co complex implies having the two handedness of the helicoidal channel within the same framework, this work represented an important milestone since it evidenced that the chirality of organometallic compounds can be transferred not only to chiral pockets or sheets in low-dimensional frameworks, as previously shown, but also to 3-dimensional open-framework materials based on helicoidal (chiral) channels. Once again, the analysis of the H-bond pattern showed this to be the origin for the transfer of chirality. However, despite their 3D open-framework structure, the presence of $\mathrm{PO}_{4}$ tetrahedra with two $\mathrm{O}$ linked to $\mathrm{Zn}$ and the other two in terminal positions probably makes this material unstable upon removal of the guest species, hence preventing its use for enantioselective operations.

The persistent effort in using chiral complexes as SDAs carried out by the group of $\mathrm{Yu}$ and coworkers finally led to the discovery of two new 4-connected chiral zeolite gallogermanate open-frameworks (JST and JSR), in this case using $\mathrm{Ni}^{2+}$ chiral complexes (cations $E$ and $D$ in Figure 6) [80,81]. GaGeO-CJ63 (JST) is a zeolitic structure with 3D intersecting 10-ring channels, built exclusively upon 3 -rings, and was synthesized using $\left[\mathrm{Ni}(\mathrm{en})_{3}\right]^{2+}$ in racemic form as the SDA (Figure 10-top) [80]. The structure is composed of cages with $C_{3}$ symmetry, which is the same as that of $\left[\mathrm{Ni}(\mathrm{en})_{3}\right]^{2+}$. As a consequence, such cages are also chiral, and indeed their chirality is related to that of the complex cations, as illustrated in Figure 10 (bottom). Molecular simulations showed that the diastereomeric complex-cage interactions found in the experimental host-guest systems $(\Lambda$-complex $\cdots \Lambda$-cage and $\Delta$-complex $\cdots \Delta$-cage) are stronger than those of the alternative diastereomeric pairs $(\Lambda$-complex $\cdots \Delta$-cage and $\Delta$-complex $\cdots \Lambda$-cage), clearly demonstrating a new example of chiral molecular host-guest recognition phenomenon, induced once again by the development of $\mathrm{H}$-bond interactions (see dashed blue lines in Figure 10-bottom). However, the use of racemic $\left[\mathrm{Ni}(\mathrm{en})_{3}\right]^{2+}$ in the synthesis involves that both enantiomers were incorporated within the framework, each of them confined within the corresponding chiral cage, and hence the overall structure was not homochiral. In a subsequent work, Yu and coworkers also prepared a new chiral zeotype gallogermanate framework (JSR) in the presence of another chiral $\mathrm{Ni}^{2+}$ complex cation, $\mathrm{Ni}$-tris(1,2-diaminopropane) $\left[\mathrm{Ni}(1,2-\mathrm{PDA})_{3}\right]^{2+}$ (cation $E$ in Figure 6) [81]. This structure contained novel chiral cavities that occurred as pairs of enantiomers containing or surrounded by the corresponding chiral enantiomers of the Ni complex, providing another example of molecular chiral recogni- 
tion between the coordination complex and particular asymmetric structural features of zeolite frameworks through the development of $\mathrm{H}$-bond interactions.

In summary, this section provides a list of examples in which the chirality of organometallic coordination compounds has been successfully transferred into oxide-based frameworks, either through chiral pockets, sheets, cages or channels. All these materials share the common feature that the impression of the chirality of the complex takes place through the development of extensive $\mathrm{H}$-bond interactions between the amino-groups ( $\mathrm{H}$-bond donor) of the ligands and $\mathrm{O}$ atoms $(\mathrm{H}$-bond acceptors) of the inorganic networks. Indeed, in the case of low-dimensional frameworks, these H-bond interactions are mainly established with the negatively-

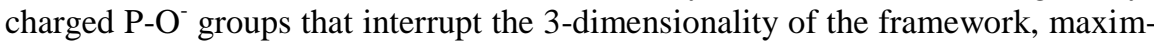
izing in this way the electrostatic interactions. In contrast, the main drawback associated to the ability of these chiral complexes of imprinting their chirality is that they establish very strong interactions with the frameworks, and as a consequence these host-guest materials are usually not stable upon removal of the chiral guest species in order to generate porosity, what has prevented so far any type of enantioselective application.

\section{Targeting known chiral zeolite frameworks: cases of study}

So far in this chapter we have dealt with the use of chiral organic or organometallic compounds in an attempt to transfer their chirality into inorganic frameworks. In this section, we change the approach to tackle the problem of searching for chiral zeolites, and we will deal with the chirality of three of the most common zeolite frameworks that are known, and make some considerations of how chiral organic SDA species could potentially transfer their asymmetric nature into them in order to produce enantiopure chiral zeolites.

\subsection{The Beta zeolite case: the quest for chiral polymorph $A$}

Zeolite Beta (*BEA) was first synthesized by Mobil researchers in 1967 using tetraethylammonium hydroxide as the organic SDA [87]. However, it was not until many years later that its structure was solved independently by Newsam et al [88] and by Higgins et al [89] in 1988. Zeolite Beta is a heavy intergrowth of two distinct but closely related polymorphs, the so-called polymorph A and polymorph B, typically in a ratio of 44:56. Both polymorphs have fully three-dimensional pore systems based on 12-rings built upon the same layers, but they differ in the way these layers are stacked on top of each other. In polymorph A, the stacking of the layers takes place through $90^{\circ}$ rotations always in the same direction, in either a right-handed $4_{1}$ sequence (RRRR...), giving place to one enantiomer (with space group $P 4_{1} 22$ ) or in a left-handed $4_{3}$ sequence (LLLL...), giving place to the other enantiomer (with space group $P_{3} 22$ ). As a consequence, the cages in polymorph 
A are arranged in a helical fashion around a four-fold screw axis along the ' $c$ ' direction, resulting in a helical channel which can correspondingly fold in a right- or left-handed manner, giving place to a chiral framework which can occur as the two possible enantiomers [18] (see Figure 11-top). In contrast, polymorph B results of a recurrent alternation of the same sheets stacked through $90^{\circ}$ rotations of the layers in right- and left-handed fashions (with a sheet stacking sequence of RLRLRL...) giving place to an achiral framework (space group $C 2 / c$ ) (see Figure 11-middle). The underlying problem here is that both polymorphs are in principle equally stable, and hence they occur with almost equal probability in zeolite beta, thus giving place to a highly faulted intergrowth of the two polymorphs. Several other stacking sequences generating other polymorphs have been proposed and some of them synthesized [90].

The discovery of the structure of zeolite Beta triggered a quest for enriching this framework in the chiral polymorph A, if possible in a homochiral (or enantioenriched form). Davis and Lobo were the first to report a zeolite beta with enantioselective catalytic properties (in the epoxide aperture of trans-stilbene oxide) by using a chiral SDA, although details were not given [91]; this demonstrated the possibility of the chiral polymorph A of zeolite beta to transfer its chiral nature into a particular chemical process of enantioselective adsorption or catalysis. Molecular simulations showed that a good match of the chiral pore geometry and that of the adsorbed molecule is required for enantioselectivity to manifest [92]. Manning and coworkers claimed enantioselective adsorption properties of zeolite Beta using chiral hydrobenzoin as a probe [93], but in this case the enantioselective adsorption probably came from an enantiomeric equilibrium between the pure enantiomers previously adsorbed on zeolite Beta and the racemic (adsorption) solution until the enantiomeric composition of both became equal.

Despite the great interest in this material, there are not many reports in the literature dealing explicitly with the use of chiral SDA cations to direct the crystallization of this framework towards the chiral polymorph A in homochiral form (see Figure 1 above). Takagi and coworkers developed a new synthetic methodology of zeolite beta in acidic medium at low temperature in order to enable the presence of chiral amine or chiral rhodium complexes (as secondary SDAs) in an attempt to transfer their chirality to the beta zeolite. In fact the authors reported a slight enrichment in polymorph $\mathrm{A}$, and they claimed that this material was able to enantioselectively adsorb a chiral amine (bis( $\alpha$-methyl)benzylamine) [94]; however, the authors compared the adsorption from pure enantiomeric solutions but not from a racemic mixture. On the other hand, several reports exist about a partial enrichment of polymorph A in zeolite beta [95]. Xia and coworkers reported that the addition of $\mathrm{W}, \mathrm{Pd}$ or $\mathrm{Pt}$ to the synthesis of zeolite beta resulted in a partial enrichment in polymorph $\mathrm{A}$, which indeed showed a certain enantioselectivity (with enantiomeric excesses, ee, up to $10 \%$ ) in the hydrogenation of tiglic acid. However, the origin for this enantioselectivity (in the absence of chiral inductors during the synthesis of the zeolite) is difficult to understand [96]. Recently two groups have claimed new synthetic protocols of zeolite beta that lead to a partial enrichment of polymorph A. On the one hand, Taborda and coworkers used an agingdrying method of the starting synthesis gels (for long times) prior to the conven- 
tional hydrothermal treatment which led to enrichments in polymorph A up to 68$70 \%$ [97,98]. On the other hand, Tong and coworkers developed a similar synthesis strategy but this time using an extremely high concentration of the synthesis gels through a dramatic reduction of the water content by heating the gels at $80{ }^{\circ} \mathrm{C}$ for several days until reaching a $\mathrm{H}_{2} \mathrm{O} / \mathrm{SiO}_{2}$ ratio of 0.3 ; this also caused a partial degradation of the tetraethylammonium cations (through Hofmann elimination) to give triethylamine $[99,100]$. In fact, it was later demonstrated that the presence of the tertiary amine played a crucial role for the enrichment in polymorph A [101]. These polymorph A-enriched zeolite beta samples doped with $\mathrm{Ti}$ showed a surprising enantioselective catalytic activity in the asymmetric epoxidation of bulky alkenes ( $\beta$-methylstyrene and 1-phenyl-1-cyclohexene), giving enantiomeric excesses up to $11 \%$, even despite the absence of using any chiral precursor in the synthesis of the catalytic materials.

These special stereoselective catalytic properties of zeolite beta further prompt the interest in having a polymorph A-enriched zeolite beta enriched in turn in one of the enantiomorphic forms. If we compare the channel systems of both polymorphs $\mathrm{A}$ and $\mathrm{B}$, it can be noticed that the difference between them is on the geometry of the channels along the $c$ axis, which are helicoidal in the case of polymorph A and straight in polymorph B (see Figure 11-bottom), while channels in the other two dimensions are similar in both polymorphs. Therefore, if one wants to favor the stacking of the beta sheets in the homochiral RRRR... (or LLLL...) fashion of polymorph A, the SDA cation should be rationally designed as to i) be chiral, ii) be large enough as to interact with more than one sheet along the $c$ axis, iii) align preferably along the $c$ axis, which is the stacking direction, while preventing its alignment with the $a$ and $b$ channels, and iv) contain a particular geometric shape with a chiral feature that prompts folding in a helicoidal fashion (in one direction and preventing the opposite) so that it adsorbs on the growing surface with such a chiral folding which favors the stacking of the subsequent sheet always in one direction while avoiding the opposite. We are currently working on rationally designing this type of chiral SDA cations.

\subsection{The ITV case: searching for chiral structure-directing agents}

A new chiral germanosilicate zeolite framework, ITQ-37 (ITV), has been recently reported by Corma and coworkers [29], which was in turn the first zeolite with a channel system in the range of mesopores. ITQ-37 crystallizes in the chiral space group $P 4_{1} 32$ (or its enantiomorph $P 4_{3} 32$ ), and is built upon a single tertiary building unit $\left(\mathrm{T}_{44} \mathrm{O}_{145}(\mathrm{OH})_{7}\right)$ comprising one $\mathrm{D} 4 \mathrm{R}$ with one $\mathrm{OH}$ group, three laumontite cages, and three D4R with two $\mathrm{OH}$ groups. The 3D arrangement of these units creates a channel system with a single gyroidal surface, with these large cavities connected to others through 30-rings, giving place to a chiral framework. The organic SDA originally used for the synthesis of ITQ-37 is achiral (even despite having four stereogenic centres, but the symmetry plane in the centre of the molecule makes it an achiral meso compound, cation $A$ in Figure 12-right), and consequently the polycrystalline ITQ-37 product is expected to be a racemic mixture of the two enantiomorphs of the chiral structure $\left(\mathrm{P} 4_{1} 32\right.$ or $\left.P 4_{3} 32\right)$. 
In a systematic study about the production of extralarge-pore zeolites with increasingly larger SDA molecules, Yu and coworkers were able to produce also ITQ-37 but using a simpler ammonium achiral cation ( $B$ in Figure 12) [47,102]. On the other hand, Chen and coworkers have recently reported a new SDA trication based on imidazolium rings for the synthesis of the same structure (cation $C$ in Figure 12) [103]. Interestingly, the authors found by fluorescence spectroscopy that these organic species arranged as supramolecular dimers within the ITV framework forming a sandwich-type structure with the phenyl rings stacked through $\pi-\pi$ type interactions. However, in both cases again the achiral nature of the cations used as SDAs should lead to a mixture of the ITV enantiomorphs. Therefore, the challenge here is to find a large-enough enantiopure chiral SDA able to direct the formation of this extra-large pore zeolite and stabilize its intrinsic low framework stability (due to the very low framework density) while being able to develop strong interactions with the framework so that it can imprint its asymmetric nature on the zeolite structure. In this context, the use of supramolecular chemistry, not only through hydrophobic $\pi-\pi$ type interactions like cation $C$ (Figure 12) but also through $\mathrm{H}$-bonds, which should extend the molecular chirality into a supramolecular level, could in principle facilitate the achievement of this goal.

\subsection{The STW case: breaking the racemic conglomerate}

In 2008 the group of Zou reported the synthesis of a new chiral zeolite based on a germanosilicate network with abundance of D4R units, SU-32 (STW structure type) [30]. The structure of this framework was fascinating as it comprised a 10-ring helicoidal channel along the $c$ axis (Figure 13; the helical channel is highlighted by dashed yellow lines). STW contains a large number of D4R units, which are stabilized by the presence of Ge and fluoride, both species known to stabilize this secondary building unit. The main structural feature of this chiral framework is that it does not crystallize as intergrown polymorphs as zeolite beta, but in contrast each single crystal grows as one unique enantiomorphic form (in $\mathrm{PC}_{1} 22$ or $\mathrm{PC}_{3} 22$ space groups). Despite the chirality of this framework, the organic molecule used originally as SDA (diispropylamine, $A$ in Figure 13) was non chiral, and consequently SU-32 was a racemic conglomerate, with each crystal being chiral itself but the overall polycrystalline powder being a 50:50 mixture of the two enantiomorphic crystals. Later on, Zhang and coworkers managed to produce STW as pure germanosilicate (and also in the presence of $\mathrm{Co}$ and $\mathrm{Cu}$ ), but using $\mathrm{N}, \mathrm{N}$-diethylethylendiamine as SDA ( $B$ in Figure 13), again an achiral species [104].

Rojas and Camblor have recently managed to produce the Ge-free all-silica version of the STW framework type by using an effective imidazolium-based SDA cation (2-ethyl-1,3,4-trimethyl-imidazolium, cation $C$ in Figure 13) which provided a great stability to this framework that compensated the absence of Ge to stabilize the D4R units (stabilized instead by the presence of fluoride) [31]. Interestingly, the authors were able to unravel the location of the organic cations. Cation $\mathrm{C}$ has one ethyl group which can rotate (around the $\mathrm{CH}_{3}-\mathrm{CH}_{2}$ bond axis), giv- 
ing different conformers. The authors proposed that the conformers which are hosted in the STW cavities are indeed chiral as long as the confinement within the cavities prevents a free-rotation of the ethyl substituent so as to lock the conformations; they could therefore be considered as pseudoatropoisomers [32]. However, an interconversion of the two mirror-image conformers of cation $C$ is expected in the absence of such confinement, i.e. in the gel, so thermodynamics (both mirror-image conformers will have the same stability in solution) will ensure the presence of the two conformers in an equal proportion and hence the crystallization of each diastereomeric pair (one conformer will drive the $P 6_{1} 22$ enantiomorph and the other mirror-image conformer will drive the $\mathrm{P}_{3} 22$ enantiomorph). Hence, at the end the overall HPM-1 sample is again a racemic conglomerate (with each crystal being enantiopure), as the authors evidenced by Mueller matrix microscopy [32].

Following the successful use of substituted imidazolium-based organic cations for the synthesis of the STW structure, Schmidt et al designed computationally new imidazolium-based organic cations that optimized the interaction with the STW framework [105]. Indeed, their computational protocol predicted the pentamethyl-imidazolium derivative (cation $D$ in Figure 13) as the most efficient SDA for this framework, as was indeed confirmed experimentally.

Based on the strong structure-directing ability of such pentamethylimidazolium units for the cavities of the STW framework, Davis et al have very recently designed a new chiral bis-imidazolium SDA dication in order to enrich the chiral STW framework in one of the enantiomorphs [52]. Chirality in the STW framework is imposed by how two consecutives cavities connect, rotated by + or $60^{\circ}$, defining the two enantiomorphic crystals; therefore, in order to impose a particular STW handedness through the use of a chiral SDA, this has to interact with two consecutive cavities. Following this reasoning, the authors designed again with the aid of molecular simulations an organic SDA with two pentamethylimidazolium rings linked by an asymmetric rigid chain based on a transdisubstituted cyclopropane unit (cation $E$ in Figure 13). Simulations showed that each enantiomer should fit much more favourably within just one of the enantiomers of the STW framework. Interestingly, the use of this chiral SDA did enable an enrichment of the STW structure in one of the enantiomers. The success in enantioselectively directing the crystallization of one enantiomorph of STW is linked to the particular molecular structure of cation $E$, with two imidazolium rings that will site in two consecutive STW cavities along the helical channel, with the handedness of the channel being imposed by the chirality of the rigid transcyclopropane linker which forces the dication to fold in one direction, following one particular handedness of the helicoidal pattern in the STW framework. Moreover, the authors were also able to produce the mirror-image of the chiral SDA, which as expected led to an enrichment of the other enantiomorph of the STW framework. Remarkably, the use of these enantiomerically-enriched STW zeolites in asymmetric catalysis did lead to certain enantiomeric excesses (up to $10 \%$ ) in the asymmetric ring-opening of large epoxides (in particular 1,2-epoxyoctane) as well as to an enantioselective adsorption of small chiral alcohols (2-butanol). In order to contrast the validity of the statement that the chirality of the STW frame- 
work did promote the enantioselective behavior, the authors used also the mirrorimage enantiomerically-enriched STW material which led to mirror-image catalytic and adsorption behaviors, as expected. Therefore, this very recent fascinating work represents the first clear example of a production of an enantiomericallyenriched chiral zeolite framework through the use of rationally-designed chiral organic SDAs, which indeed behaves enantioselectively in asymmetric catalytic and adsorption processes. This successful case, after a so long quest for homochiral zeolite frameworks, will surely prompt an intensive effort to search for new enantiomerically-enriched chiral zeolite frameworks, possibly with larger pores, to enable the processing of larger chiral molecules of interest, in particular in the pharmaceutical industry.

\section{Chiral spatial distributions of dopants: a new concept of chirality in zeolites}

Except for the very recent case just reported about the enantiomerically-enriched STW zeolite [52], there has been very limited success in transferring the chirality from enantiopure organic species into homochiral open-framework zeolite structures. In this context, it is interesting to mention the work of Castillo and coworkers [106] which used molecular simulations in an attempt to understand the factors governing the transfer of chirality from chiral zeolite frameworks onto chiral sorbates (similar conclusions could be transferred in the opposite direction, applied to the imprinting of chirality from the organic SDAs to the nascent zeolite framework). In their work, the authors studied the performance of SOF, STW and ITN frameworks to discriminate between enantiomers of $\mathrm{CHBrClF}$ and 4-ethyl-4methyloctane. The authors concluded that a proper match between the pore geometry and the packing of the chiral sorbate molecules within the channel system is required for enantioselectivity to occur: a tight fitting of the chiral sorbate in the zeolite channels will favor enantiodifferentiation. Indeed, they proposed that the main reason for the observation of enantiodiscrimination is the different longrange asymmetric packing interactions between the enantiomers of the chiral sorbate. Hence, when transferred to the imprinting of the asymmetric nature of chiral organic SDAs onto zeolite frameworks, a proper match of the molecular shape and that of the zeolite framework is as important as the occurrence of lateral asymmetric long-range packing interactions of the SDA species embedded in the zeolite channels.

On the other hand, another possibility for the lack of transfer of chirality is related to the high mobility that is usually associated with the SDAs (which are the chiral carriers) confined within the microporous systems. This is caused by the typical lack of a tight fitting of the SDAs and the surrounding pores/cavities [17]. Such high mobility involves that the SDAs can freely rotate during the crystallization process, what would dissipate their asymmetric feature. The failure in transferring the chirality is also explained by the typically weak and non-directional in- 
teractions established between the terminal alkyl groups of the organic SDA species and the oxide zeolite networks. Section 3 has clearly evidenced that the transfer of chirality is notably enhanced with the use of chiral coordination complexes based on polyamine ligands, which have abundant primary amino groups which develop strong $\mathrm{H}$-bond interactions with the $\mathrm{O}$ atoms of the framework, and especially with the negatively-charged $\mathrm{O}$ atoms (in terminating $\mathrm{PO}^{-}$groups). However, the main drawback for the use of these chiral complexes as SDAs is that so far they have led to frameworks which are not stable upon removal of the guest species, possibly a consequence of those strong H-bond interactions that stabilize the systems.

Another likely cause for the failed transfer of chirality might come from the different dimension of chirality between the host and guest species, in which chirality from the organic species are typically at an atomic (or molecular) level, thus generating an asymmetric environment at a local (short-ranged) level, compared to the chirality of zeolite structures which is associated to a long-range chiral ordering (commonly in the form of helical channels) of the achiral $\mathrm{TO}_{4}$ units. In this line, if we want to optimize the transfer of chirality from the organic molecules, we need to expand their chirality into a larger level so that the dimensions of both chiral features are adapted to each other. In fact, this was the main molecular feature of cation $E$ (Figure 13) that enabled the transfer of chirality onto the STW framework: the use of large dications with a chiral spacer in the middle which spanned two consecutive cavities of the chiral framework, with the asymmetric nature of the spacer favoring only one of the enantiomorphic frameworks.

Based on all these considerations, we have proposed in our group a new strategy to induce chirality in zeolite frameworks. In order to transfer their chirality onto zeolite frameworks, chiral SDAs should i) expand their asymmetric nature into the long-range, possibly through supramolecular asymmetric packing interactions, in order to adapt the host/guest chiral dimensions, ii) their motion should be restricted in order to enhance the manifestation of the asymmetric environment, and iii) they should establish strong H-bond interactions with the zeolite framework.

In an attempt to expand their asymmetric nature into a long-range level, it becomes crucial to change the traditional concept of isolated organic SDA species, whose structure-directing effect takes place through single molecular units, thus generating chiral microenvironments at a local level, into self-assembling entities which can develop supramolecular chiral entities stabilized by intermolecular interactions, thus extending the chiral feature into the supramolecular level. An easy way of achieving this self-assembly in aqueous systems is by using chiral molecules with aromatic rings, since these tend to form supramolecular aggregates in water stabilized by $\pi-\pi$ interactions between the aromatic rings (Figure 14-top), as explained in detail in Chapter 5 in this Volume. The formation of these supramolecular aggregates involves a first extension of the chiral environment to the supramolecular level. In turn, the formation of these dimers will notably reduce the motion of the SDAs when confined within zeolite pore systems. Moreover, in the case of being confined in zeolite systems based on channels, the molecular chirality also involves that the lateral or packing interactions between consecutive dimers will also be asymmetric, with one particular fitting (in turn a particular rota- 
tion between consecutive dimers around the channel axis) being favoured, thus developing a supramolecular helicoidal arrangement of the dimers (Figure 14-top), with one rotation being favored with respect to the opposite (mirror-image) one, and hence developing homochiral supramolecular entities with a long-range chiral dimension that could be potentially transferred into the zeolite framework.

Apart from this strategy, in order to maximize our chances of transferring chirality, we still need to enhance the interactions between the zeolite frameworks and a localized area of the organic SDAs, preferably associated to the stereogenic centre so that the chiral feature is more strongly manifested. As previously mentioned, the most successful chiral SDAs used so far are chiral coordination complexes which imprint their chirality by the establishment of extensive H-bond interactions through primary amine groups. Therefore, it would be convenient to use amines which can develop H-bond interactions (rather than quaternary ammonium cations). In this context, amines are better SDAs for the synthesis of AlPO-based (rather than silica-based) zeolitic frameworks due to the neutral-to-acidic pHs at which their synthesis is performed, which enables a protonation of the amines and hence a development of strong interactions with the network. On the other hand, the strongest and more localized interactions during a zeolite crystallization process are established between the positive charge associated to the protonated ammonium groups of the organic molecules and the negative charge associated to defects in the zeolite network, commonly in the form of low-valence isomorphic substitutions of $\mathrm{Si}^{4+}$ by $\mathrm{Al}^{3+}$ in zeolites, or $\mathrm{Al}^{3+}$ by divalent metals like $\mathrm{Mg}^{2+}, \mathrm{Zn}^{2+}$ or $\mathrm{Co}^{2+}$ in AlPO networks. In this context, the dopants in the zeolitic networks can adopt a large number of different spatial configurations depending on the available $\mathrm{T}$ positions where the dopant is to be incorporated (Figure 14-bottom, blue balls). This spatial distribution of dopants arises a new degree of freedom and consequently provides a matrix where a chiral feature could be potentially imprinted, in the form of asymmetric distributions of dopants embedded in otherwise achiral frameworks (Figure 14-bottom, green balls), which represents a new concept of chirality in zeolite materials that we have recently proposed [107-109]. In fact, the strong interactions established between the positive charge of protonated ammonium groups containing $\mathrm{H}$-bond donor groups and the negative charge associated to the incorporation of dopants make possible that these organic species can direct the spatial incorporation of dopants, as we have demonstrated in other FER systems $[110,111]$. Therefore, it would in principle be feasible that if we manage to promote such supramolecular helicoidal arrangements of the organic aromatic SDA molecules through asymmetric packing interactions, and these aromatic amines (or rather protonated ammonium cations) develop strong electrostatic (and H-bond) interactions with the dopants, such helicoidal organic arrangement could be imprinted on the zeolite network through a helicoidal spatial distribution of dopants, hence inducing chirality in the zeolite framework. Interestingly, Song and coworkers have discovered a new zeolite framework (JRY) where a helix of cobalt atoms occurs within the framework, leading to a chiral material [28]. In fact, despite using achiral ethylamine as SDA, circular dichroism experiments did surprisingly reveal a certain enantiomeric excess in the solid material, which was ascribed to a symmetry breaking event amplified by secondary nucleation. 
Following this reasoning, we designed a chiral (enantiopure) aromatic amine as SDA derived from $L$-proline, $(S)$-(-)-N-benzyl-pyrrolidine-2-methanol (BPM) $[59,107]$ (molecule $Q$ in Figure 3), which directed the crystallization of the AFI AlPO-based framework in the presence of different dopants. Interestingly, molecular mechanics simulations showed that the most stable packing of dimers of BPM along the AFI channels involved an asymmetric supramolecular arrangement where consecutive dimers were always rotated by an angle of $-90^{\circ}$ (this represented the most stable rotation angle, see Figure 15-top-left, blue line), thus leading to a helicoidal supramolecular arrangement of the organic SDA species, which was our initial target (Figure 15-top-right) [107]. Of course the occurrence of just one particular rotation angle being more stable than the rest (and especially than the mirror-image rotation angle in the opposite direction) was a direct consequence of the introduction of the hydroxymethyl substituent in the pyrrolidine ring that made the molecule chiral. This was evidenced by the fact that rotations between consecutive dimers in one or the opposite direction for the achiral equivalent molecule, benzylpyrrolidine, were equally stable (see Figure 15-top-left, red line). However, fluorescence spectroscopy results showed that the actual incorporation of these BPM molecules confined within the AFI 1-dimensional channels involved both monomer and dimer species, with relative amounts of each depending on the type of dopant and the protonation state of the SDA: protonated dimers seem to cause an electrostatic repulsion between consecutive dimers that disfavored the occlusion of dimers [112,113]. The non-exclusive incorporation of dimers would of course disrupt the supramolecular helicoidal arrangement of the dimers. Moreover, we observed that the interaction between the ammonium groups of BPM dimers and the divalent dopants located in the different positions (replacing $\mathrm{Al}$ in the channel walls) was weak since these ammonium groups sited close to the centre of the channels and hence away from the channel walls (with distances larger than $5 \AA$ ), and hence they could not interact with the dopants. Therefore, it did not seem likely that these BPM dimers would direct the spatial incorporation of dopants, as evidenced by the fact that similar stabilities were found for AFI/BPM systems with $\mathrm{Mg}$ sited in different $\mathrm{Al}$ positions (see Figure 15-bottom-left). As a consequence, a transfer of chirality from the supramolecular dimer arrangement into a helicoidal distribution of dopants did not seem likely to occur for this particular chiral SDA molecule.

We then selected another molecule, $(1 R, 2 S)$-ephedrine (EPH, molecule $R$ in Figure 3), that satisfied the same requirements previously commented, while being potentially able to interact more-strongly with the framework walls through $\mathrm{H}$ bond interactions. The selection of this chiral precursor was based on the simultaneous occurrence of i) an aromatic ring which promotes the formation of supramolecular dimers through $\pi-\pi$ type interactions, ii) two stereogenic centers that impart a strong asymmetric molecular structure, iii) H-bond donor and acceptor groups that trigger an asymmetric packing (lateral) interaction between consecutive dimers, potentially leading to long-range chiral orderings, and iv) a secondary amine which will be protonated in acidic medium to form $\mathrm{R}-\mathrm{NH}_{2}{ }^{+}-\mathrm{R}^{\prime}$ positivelycharged groups that will develop strong electrostatic interactions with the negative charge associated to divalent dopants. As previously mentioned, the use of this 
molecule as SDA for the synthesis of nanoporous aluminophosphates led to the crystallization of the AFI framework [61,62]. Molecular mechanics simulations then showed the occurrence of one particular rotation between consecutive dimers which was more stable than the others $\left(+30^{\circ}\right.$, see Figure 16-top-left), and hence consecutive dimers will tend to be rotated by this angle, developing again a helicoidal supramolecular arrangement of EPH dimers (Figure 16-top-right) [114]. This particular rotation represented a more stable packing configuration since it enabled the development of a stronger double $\mathrm{H}$-bond interaction between the $\mathrm{NH}_{2}{ }^{+}$and $\mathrm{O}(\mathrm{H})$ groups of consecutive dimers (Figure 16-bottom-left). Interestingly, in this case the $\mathrm{NH}_{2}{ }^{+}$did locate close to the channel walls and did interact strongly with them. In fact, there was a clear relationship between the stability of the different locations of the divalent dopants with their distance to the ammonium group (see Figure 15-bottom-right): the dopants prefer to locate close to the ammonium positive charge. This is an indication that the ammonium groups will direct the spatial distribution of dopants in the AFI channels, and hence suggests that the helicoidal supramolecular arrangement of the EPH dimers could potentially be transferred into a helicoidal spatial distribution of dopants (Figure 16-bottomright).

Nonetheless, although we have clearly demonstrated the exclusive incorporation of ephedrine dimers within the AFI framework (depending on the synthesis conditions) $[68,69]$, so far we have not been able to experimentally demonstrate the occurrence of the chiral distribution of dopants. Confirmation of such special configuration for the dopants is extremely difficult (if not impossible) by diffraction techniques (either electron or X-Ray diffraction methods) due to the similar scattering power of $\mathrm{Mg}$ dopants and $\mathrm{Al}$ and by the subtle changes that would be induced in the diffraction pattern as a consequence of these particular dopant distributions. Therefore, in an attempt to demonstrate the occurrence of this concept of chirality and its potential use in enantioselective operations, we are currently working on the adsorption of different chiral probe molecules within these materials.

\section{Summary}

In this chapter we have analyzed in detail the potential of transferring chirality into zeolite frameworks through the use of chiral structure-directing agents. First attempts by using chiral organic species led invariably to a failure in imprinting their chirality onto zeolite networks, possibly because of a lack of strong and specific host-guest interactions, high mobility of the chiral precursors within the channels and/or cavity systems, and the different dimension of the chiral feature in the organic unit (at a local environment) and in the zeolite framework (at a longrange level).

The use of chiral organometallic complexes has been more successful in transferring their chirality onto inorganic frameworks through the establishment of ex- 
tensive H-bond interactions; however they usually promoted the crystallization of low-dimensional frameworks, or 3D zeolitic open-frameworks but where the guest species are removed with difficulty and probably causing a collapse of the structure. Nevertheless, these works clearly demonstrate the fundamental role of $\mathrm{H}$ bond interactions when imprinting chirality on an inorganic lattice.

There has been a very recent example where a computationally-guided rational design of a chiral SDA enabled the production of an enantiomerically-enriched chiral zeolite framework, which represents a fundamental milestone in the quest for a homochiral zeolite structure, a highly desired yet elusive target in zeolite science. This work not only demonstrates the feasibility of producing homochiral zeolite materials where chirality is imprinted through the use of rationally-selected chiral SDAs, where the aid of molecular simulation techniques has proven fundamental, but also confirms that this enantioenriched chiral zeolite materials are able to perform enantioselective operations of both asymmetric catalysis and adsorption (although so far the enantiomeric excesses found are not very high). Hence, this fundamental work will probably prompt a more intensive search for other chiral zeolite frameworks in order to produce active, robust and efficient enantioselective catalysts, which will potentially be of enormous relevance in the chemical industry, especially in the pharmaceutical sector.

Acknowledgements. Funding from the Spanish Ministry of Science and Innovation (MICINN) through projects MAT2012-31127 and MAT2015-65767-P is acknowledged. BBM acknowledges the Spanish Ministry of Economy and Competitivity for a predoctoral (BES-2013-064605) contract. 


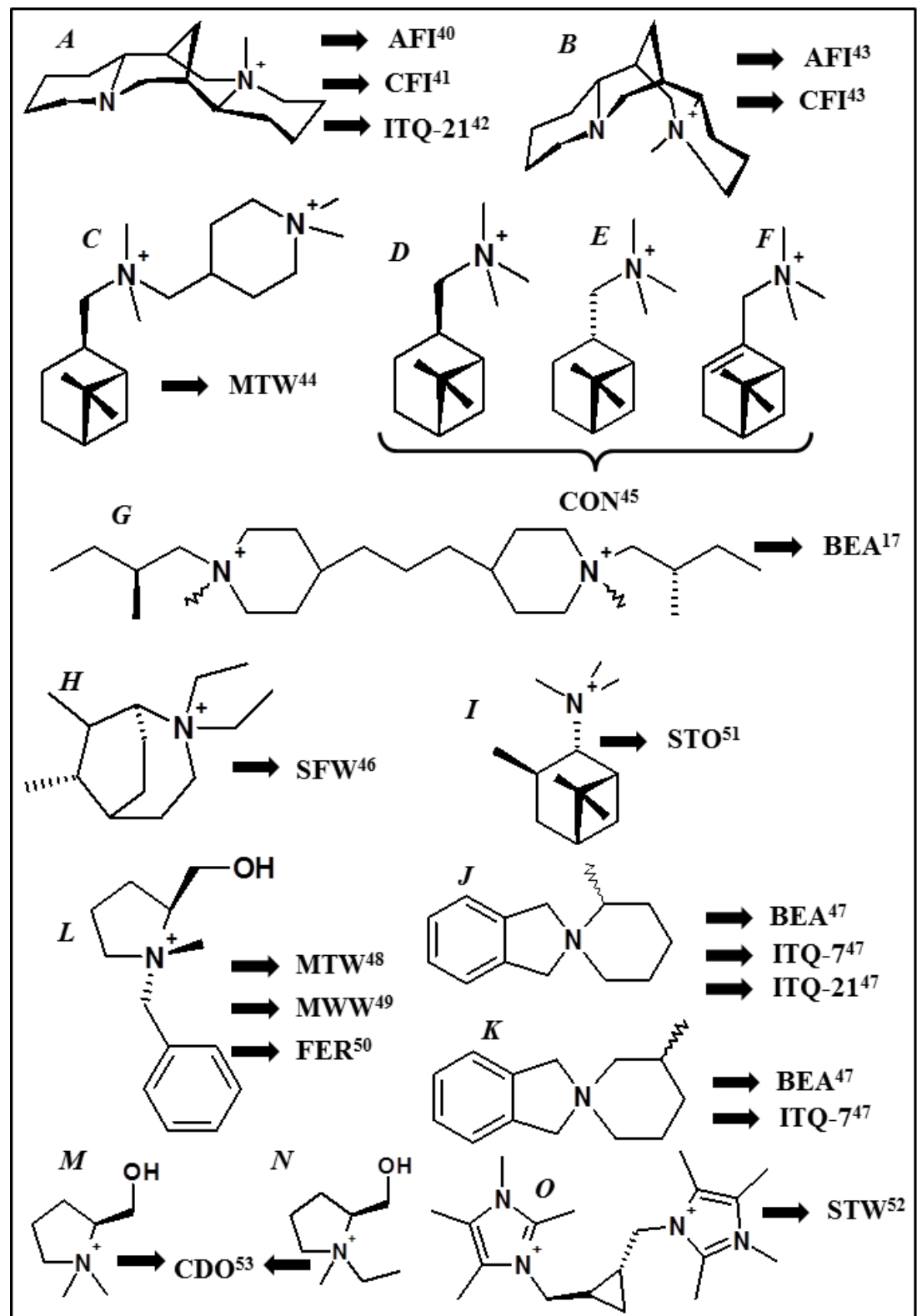

Figure 1. Chiral organic cations used as structure-directing agents that directed the crystallization of zeolite frameworks (silica-based composition). 


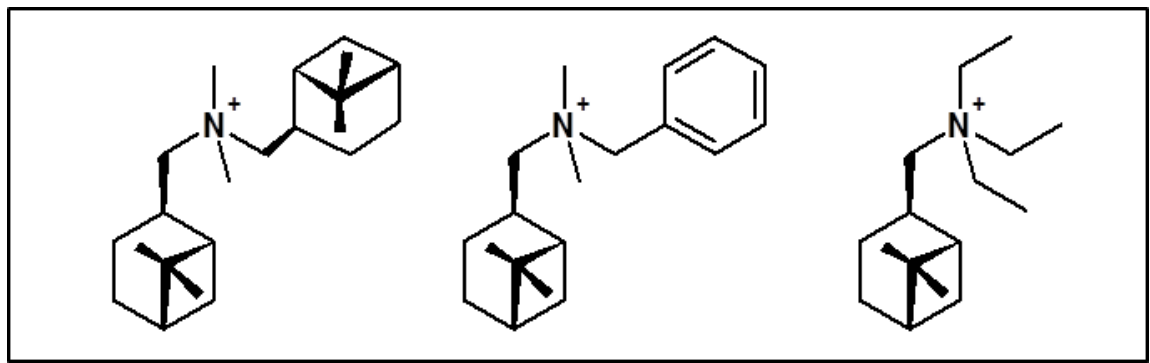

Figure 2. Additional chiral organic cations derived from the myrtanyl ring used as SDAs that did not lead to crystalline zeolite materials (in silica-based compositions) [44]. 


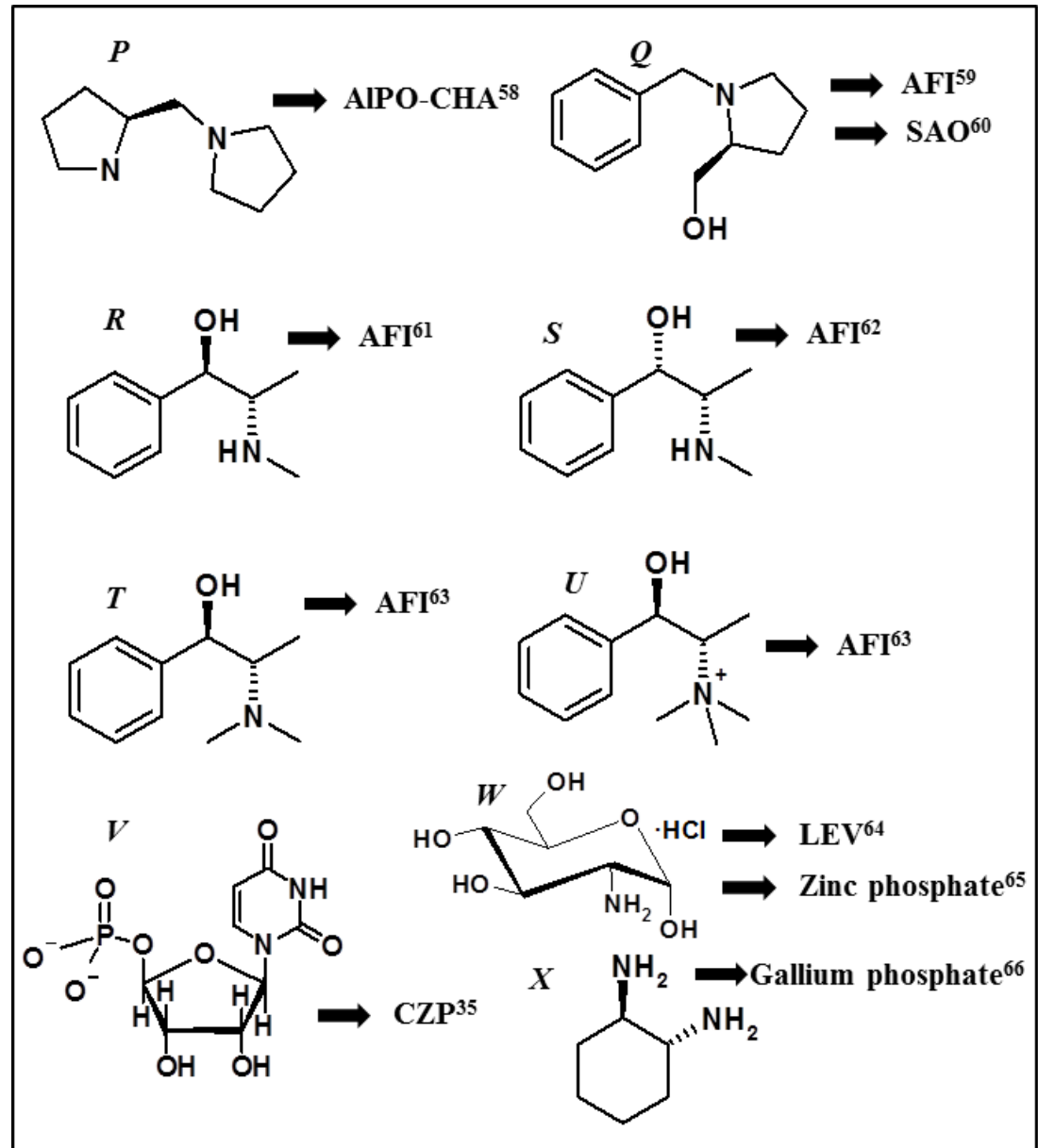

Figure 3. Chiral organic molecules used as structure-directing agents that directed the crystallization of zeotype frameworks (AlPO, ZnPO or GaPO-based compositions). 

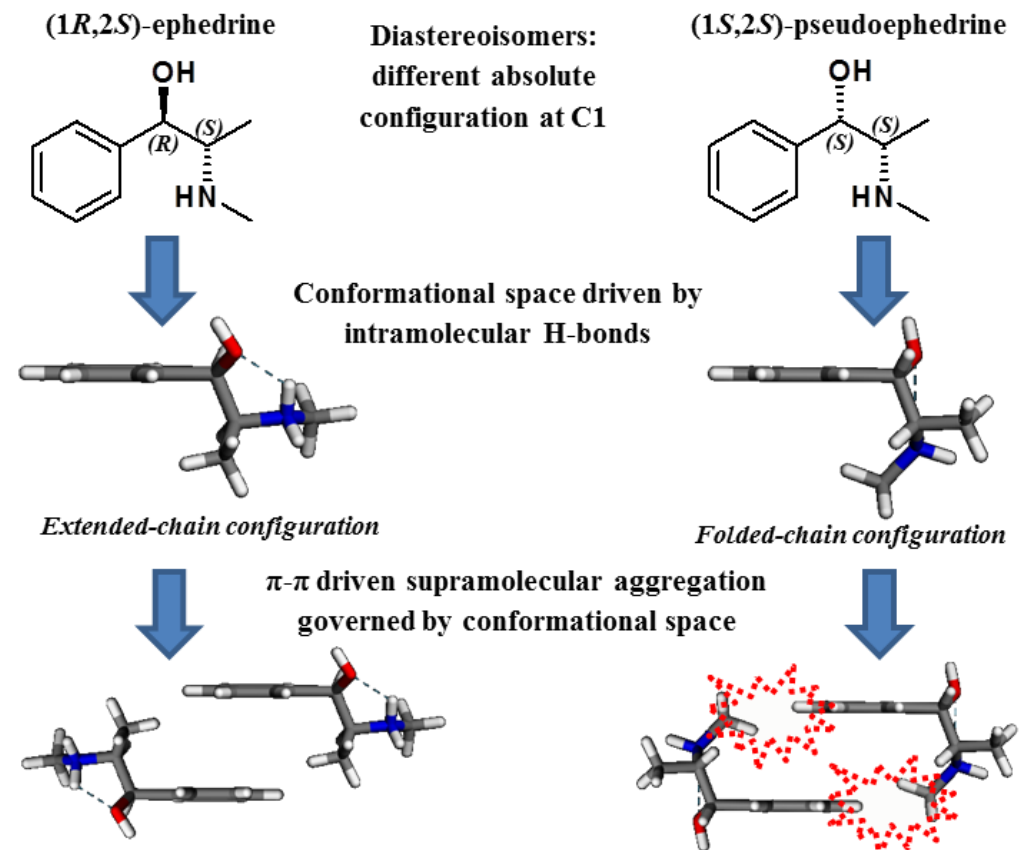

Strong supramolecular aggregation

Folded-chain configuration

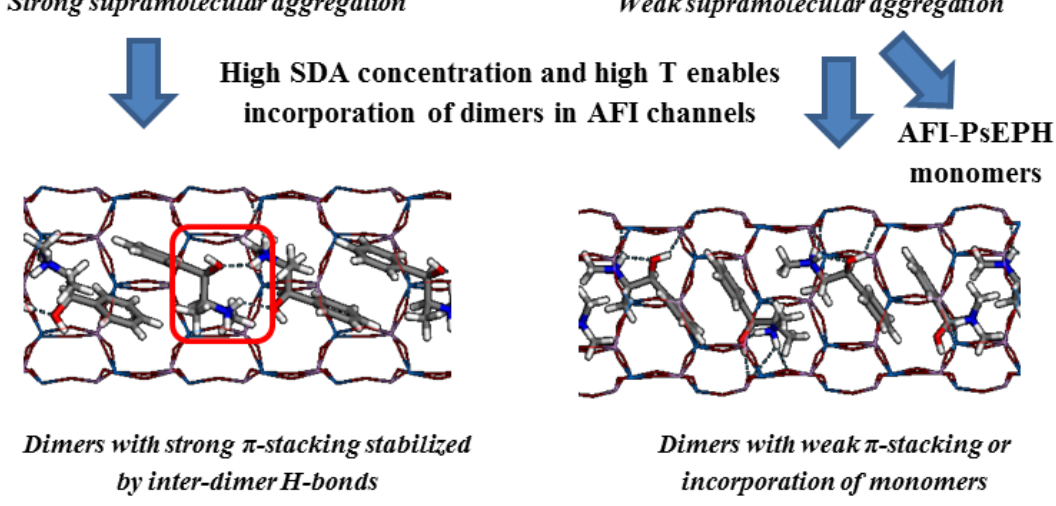

Figure 4. Schematic picture with the structure-directing effect of $(1 R, 2 S)$ ephedrine (left) and $(1 S, 2 S)$-pseudoephedrine (right) and their associated supramolecular chemistry during occlusion within the AFI channels. Adapted with permission from [62], Copyright (2015) American Chemical Society. 


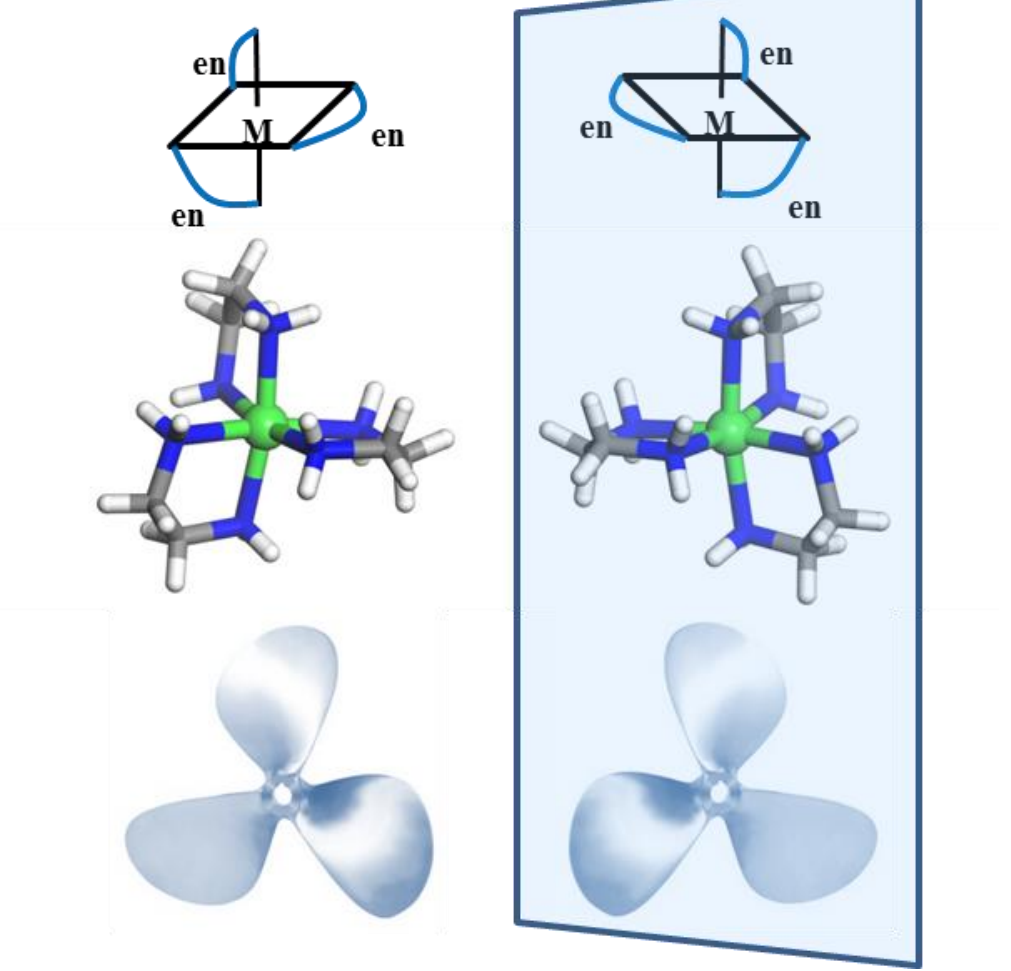

Figure 5. Schematic picture of the propeller chirality of octahedral transitionmetal coordination compounds with bidentate ligands. 


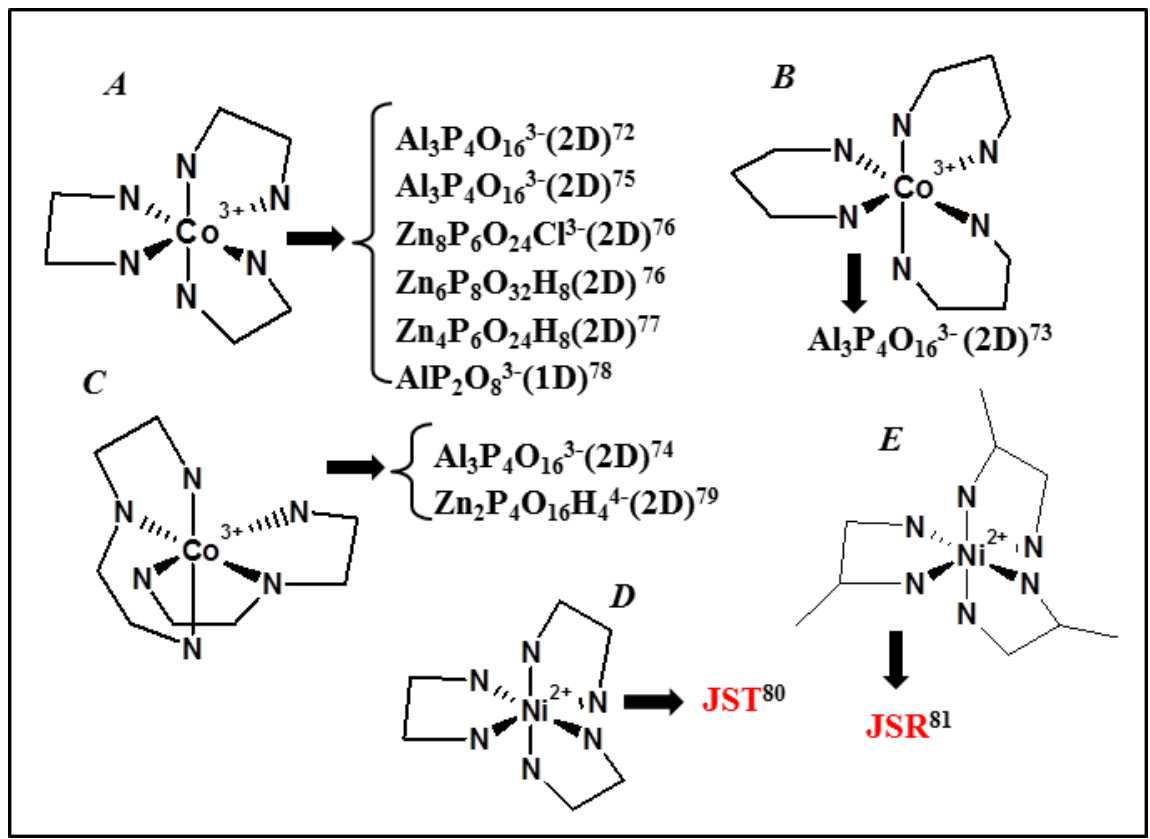

Figure 6. Some of the most common chiral organometallic coordination complexes used as SDAs for the crystallization of low-dimensional or zeotype frameworks (AlPO, $\mathrm{ZnPO}$ or GaGeO-based compositions); chiral zeolite frameworks are shown in red. 

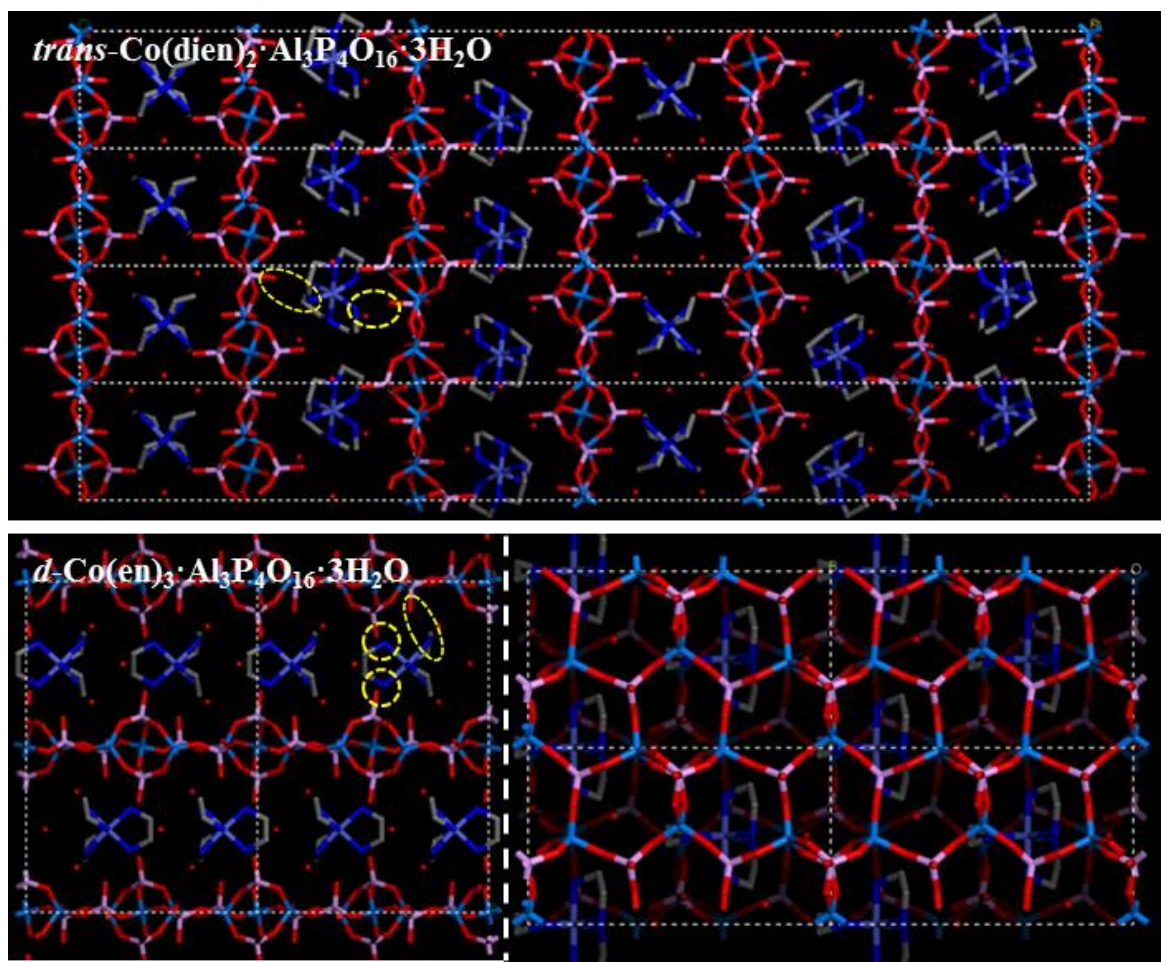

Figure 7. Structure of trans-Co(dien) $)_{2} \cdot \mathrm{Al}_{3} \mathrm{P}_{4} \mathrm{O}_{16} \cdot 3 \mathrm{H}_{2} \mathrm{O}$ (top) [74] and of $d$ $\mathrm{Co}(\mathrm{en})_{3} \cdot \mathrm{Al}_{3} \mathrm{P}_{4} \mathrm{O}_{16} \cdot 3 \mathrm{H}_{2} \mathrm{O}$ (bottom) [75] (H atoms are not shown). $\mathrm{O}$ atoms are shown in red, $\mathrm{Al}$ in blue, $\mathrm{P}$ in pink, $\mathrm{N}$ in dark blue, $\mathrm{Co}$ in light blue and $\mathrm{C}$ in grey. 

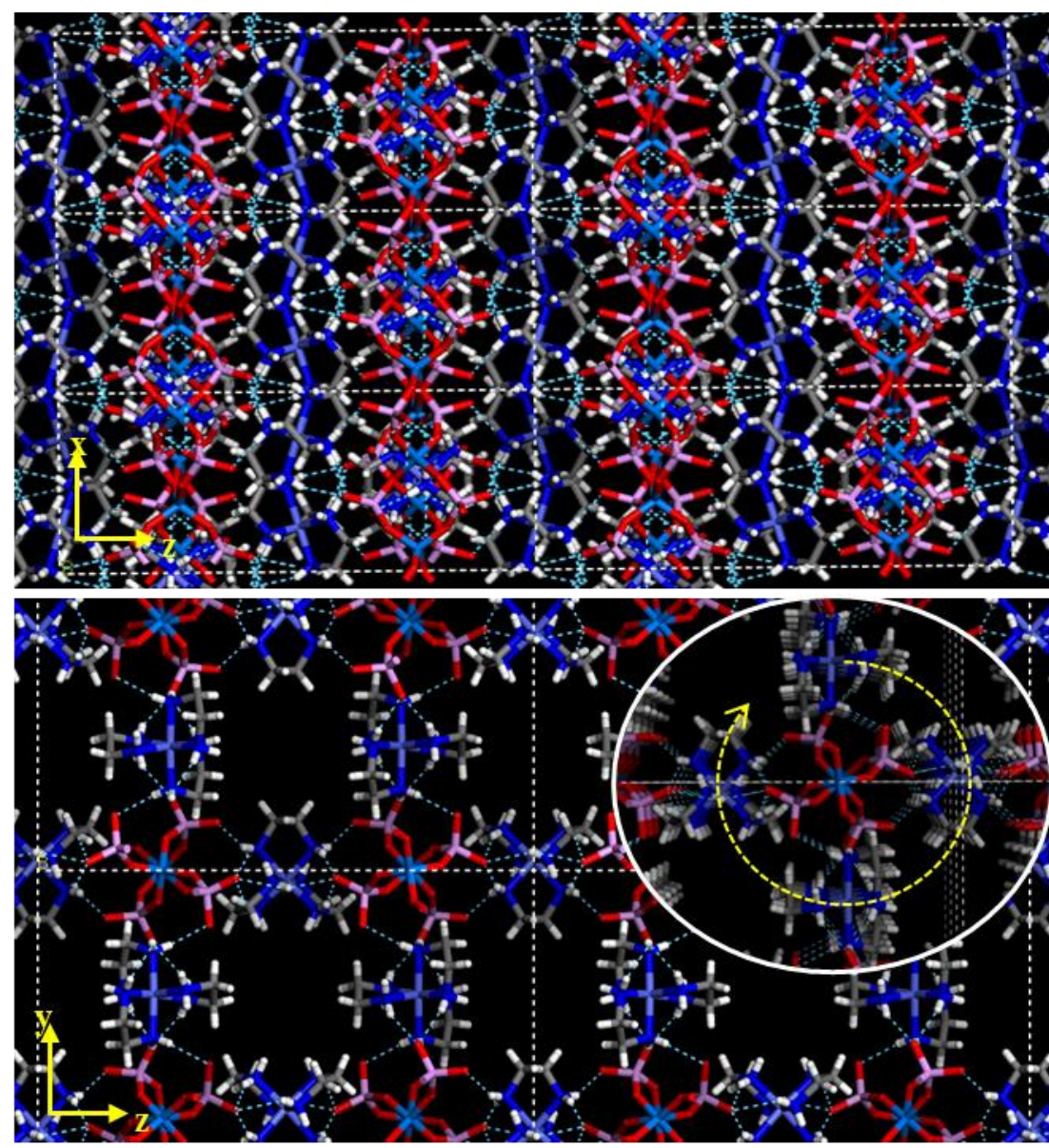

Figure 8. Two views (top and bottom) of the structure of $d$ -

$\mathrm{Co}(\mathrm{en})_{3} \cdot \mathrm{AlP}_{2} \mathrm{O}_{8} \cdot 6.5 \mathrm{H}_{2} \mathrm{O}$, highlighting the $\mathrm{H}$-bond helix around the AlPO chain

[78]. $\mathrm{O}$ atoms are shown in red, $\mathrm{Al}$ in blue, $\mathrm{P}$ in pink, $\mathrm{N}$ in dark blue, $\mathrm{Co}$ in light blue, $\mathrm{C}$ in grey, and $\mathrm{H}$ in white. Dashed blue line indicates $\mathrm{H}$-bonds. 

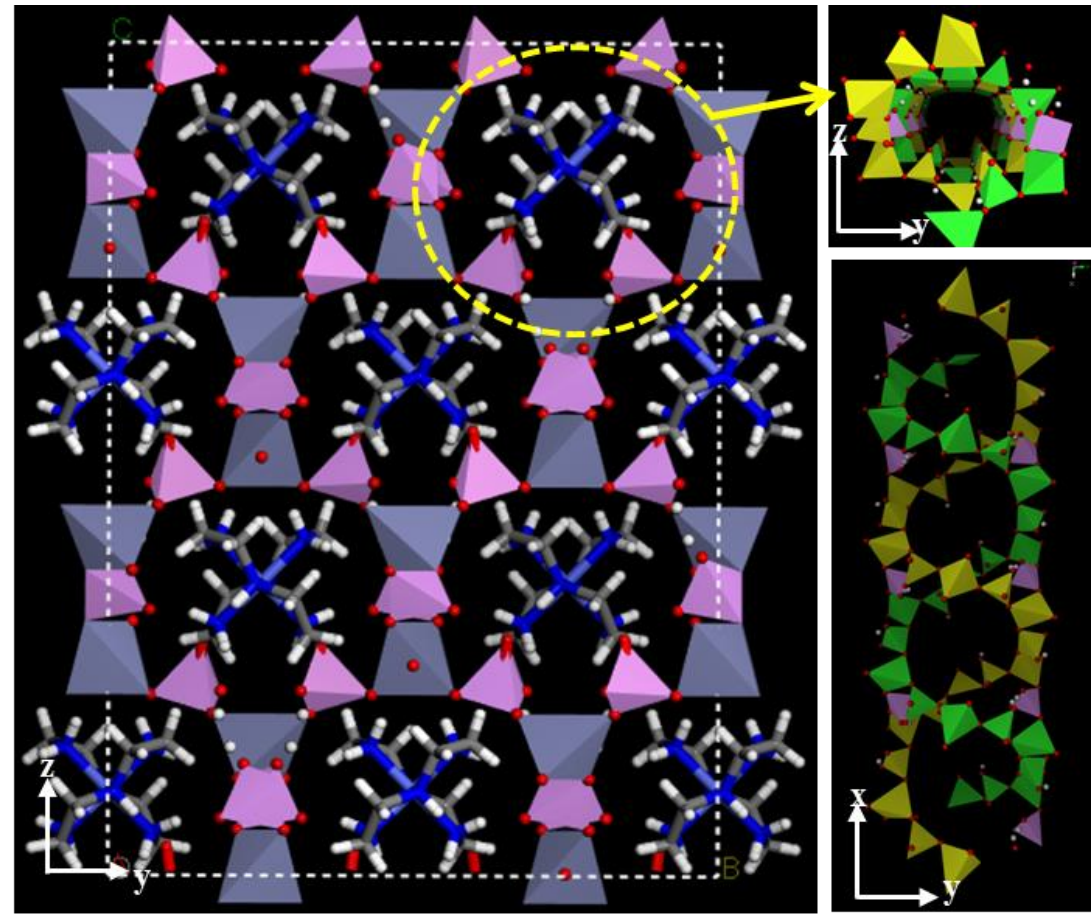

Figure 9. Structure of $\left(\left[\mathrm{Zn}_{2}\left(\mathrm{HPO}_{4}\right)_{4}\right]\left[\mathrm{Co}(\text { dien })_{2}\right] \cdot \mathrm{H}_{3} \mathrm{O}\right.$ (left), highlighting the helicoidal 12-ring channel with the two strands in different colors (right) [79]. O atoms are shown in red, $\mathrm{Zn}$ and $\mathrm{P}$ as grey and pink polyhedra, $\mathrm{N}$ in dark blue, Co in light blue, $\mathrm{C}$ in grey, and $\mathrm{H}$ in white. The two intertwined helices are highlighted as yellow and green polyhedra (right). Adapted with permission from [79], Copyright (2003) John Wiley and Sons. 


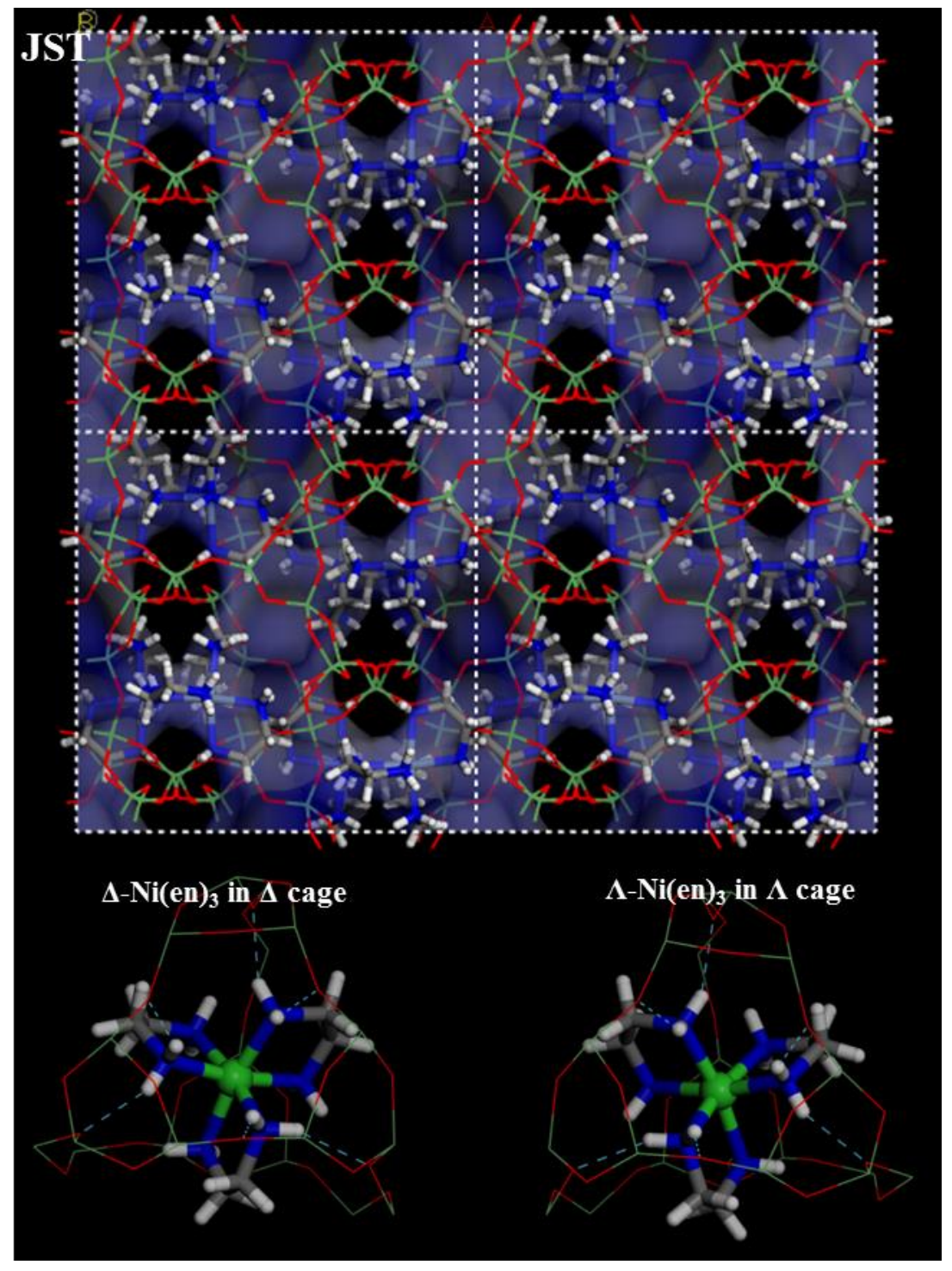

Figure 10. Structure of JST structure type (GaGeO-CJ63) (top) and stereochemical relationship between the chirality of the complex cations and that of the cages $(\mathrm{H}-$ bond interactions are indicated by dashed blue lines) [80]. The free volume is shown as a blue surface (top). $\mathrm{O}$ atoms are displayed in red, $\mathrm{Ga} / \mathrm{Ge}$ in dark green, $\mathrm{N}$ in blue, $\mathrm{C}$ in grey, $\mathrm{H}$ in white and $\mathrm{Ni}$ in light green. Adapted with permission from [80], Copyright (2011) John Wiley and Sons. 

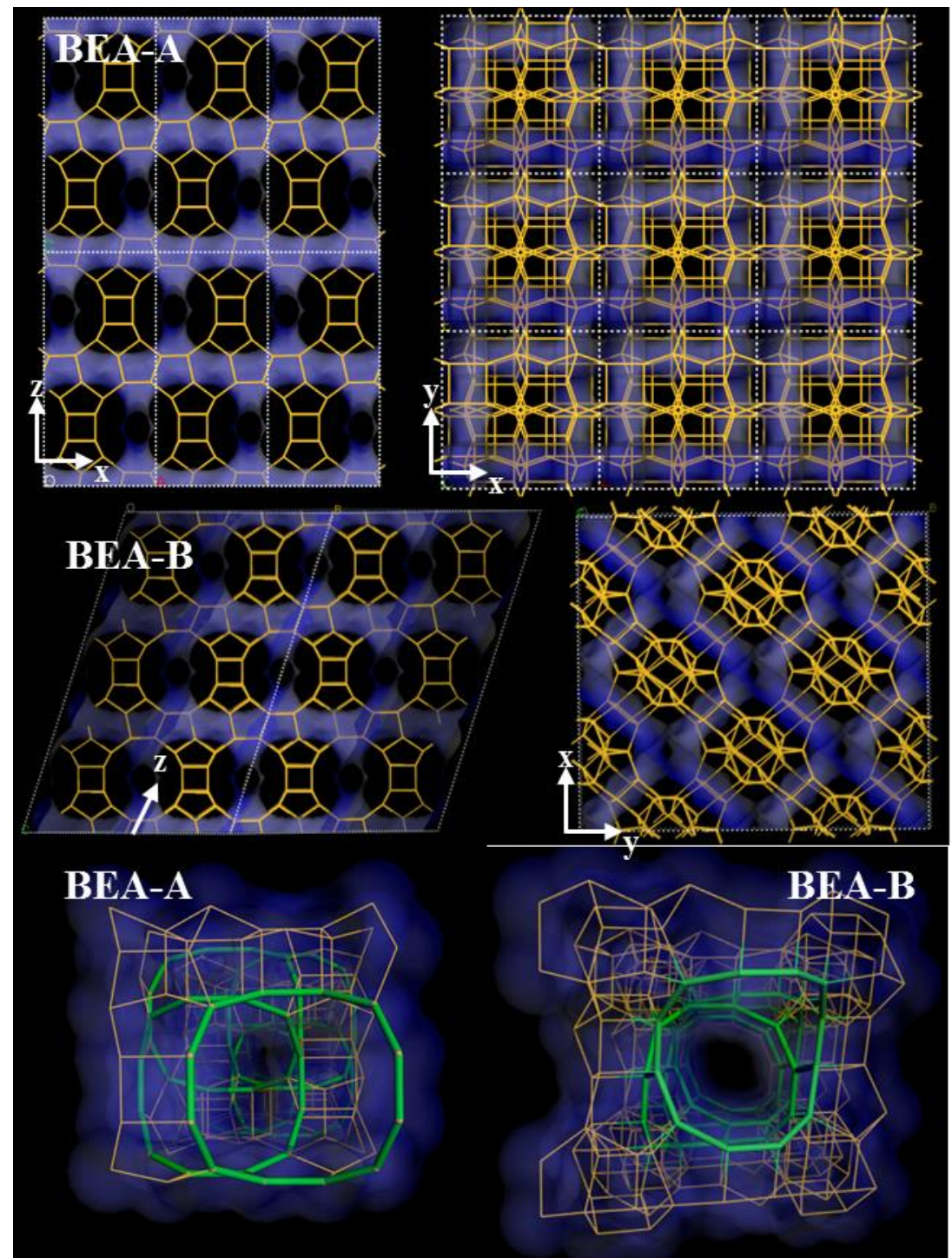

Figure 11. Different stacking of the Beta sheets giving place to polymorph A (top) or B (middle), and generation of the distinct channels in the ' $c$ ' direction (bottom). 

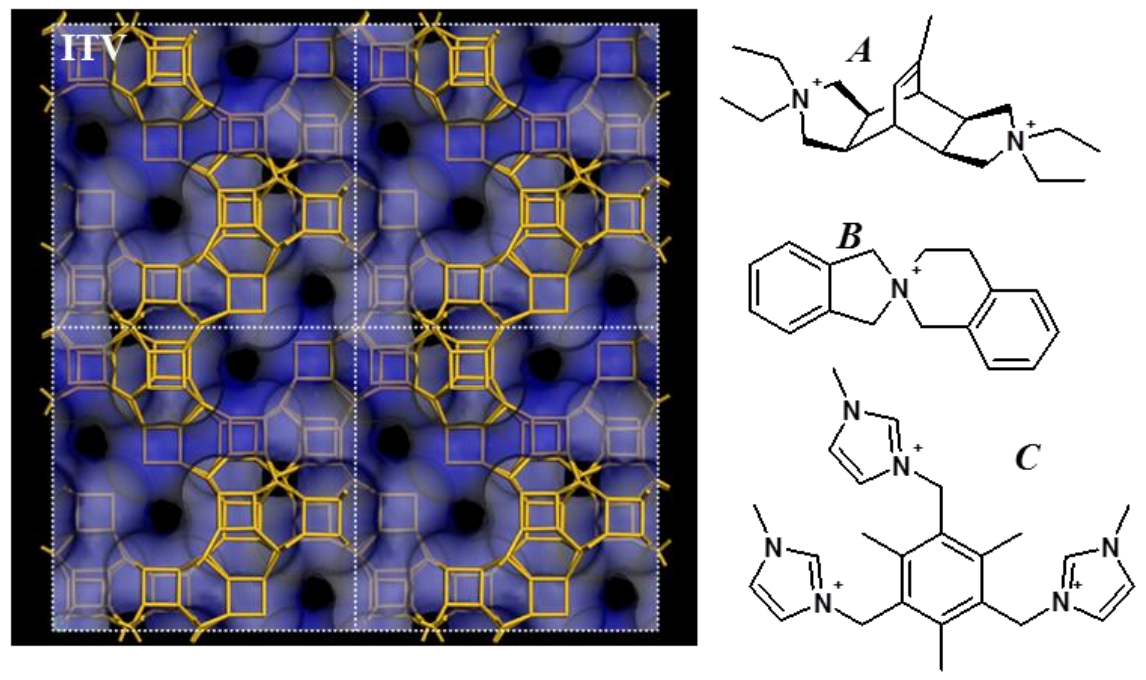

Figure 12. The ITQ-37 framework structure (ITV) (left), and the organic cations used as SDAs that have directed its crystallization so far (right). 


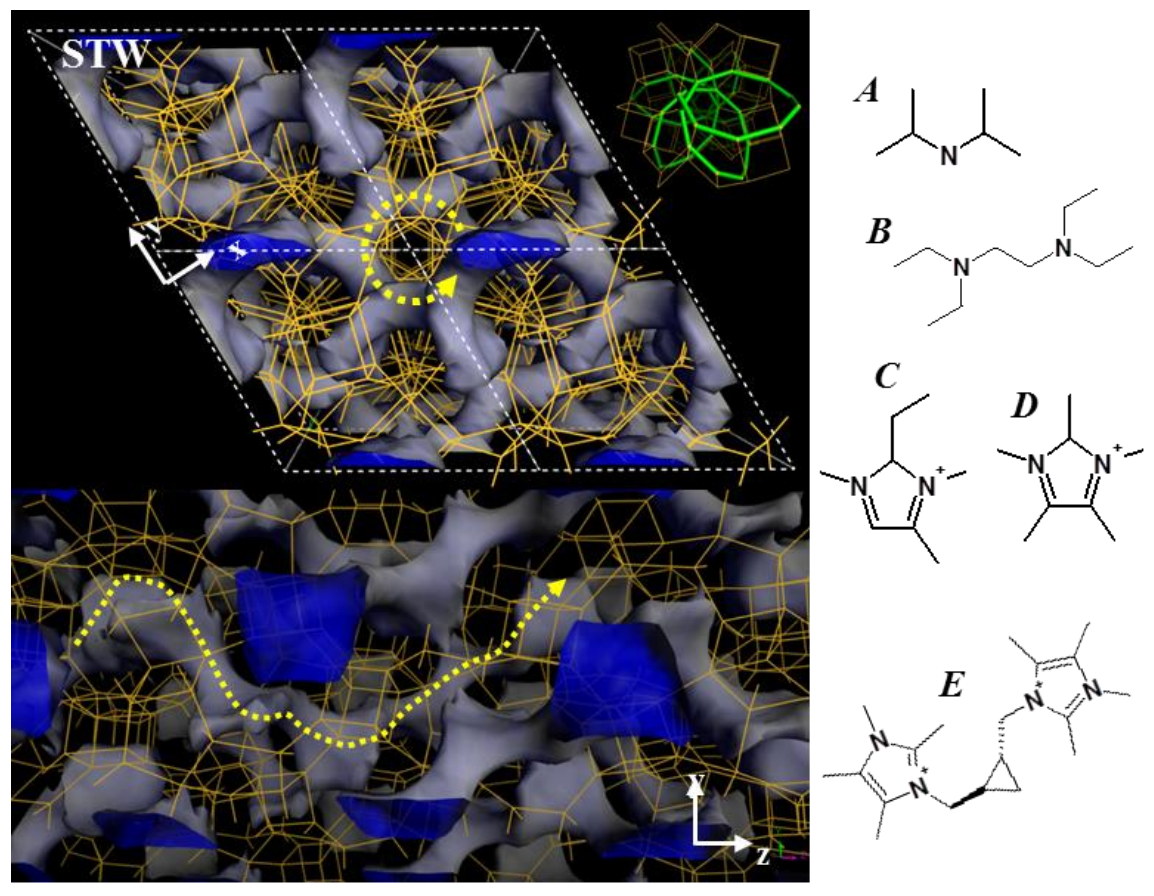

Figure 13. The STW framework structure (left), with details of the helical channel (highlighted by yellow dashed lines; a detail of the channel is highlighted by green sticks at top-right), and the organic species used as SDAs that have directed its crystallization so far (right). 


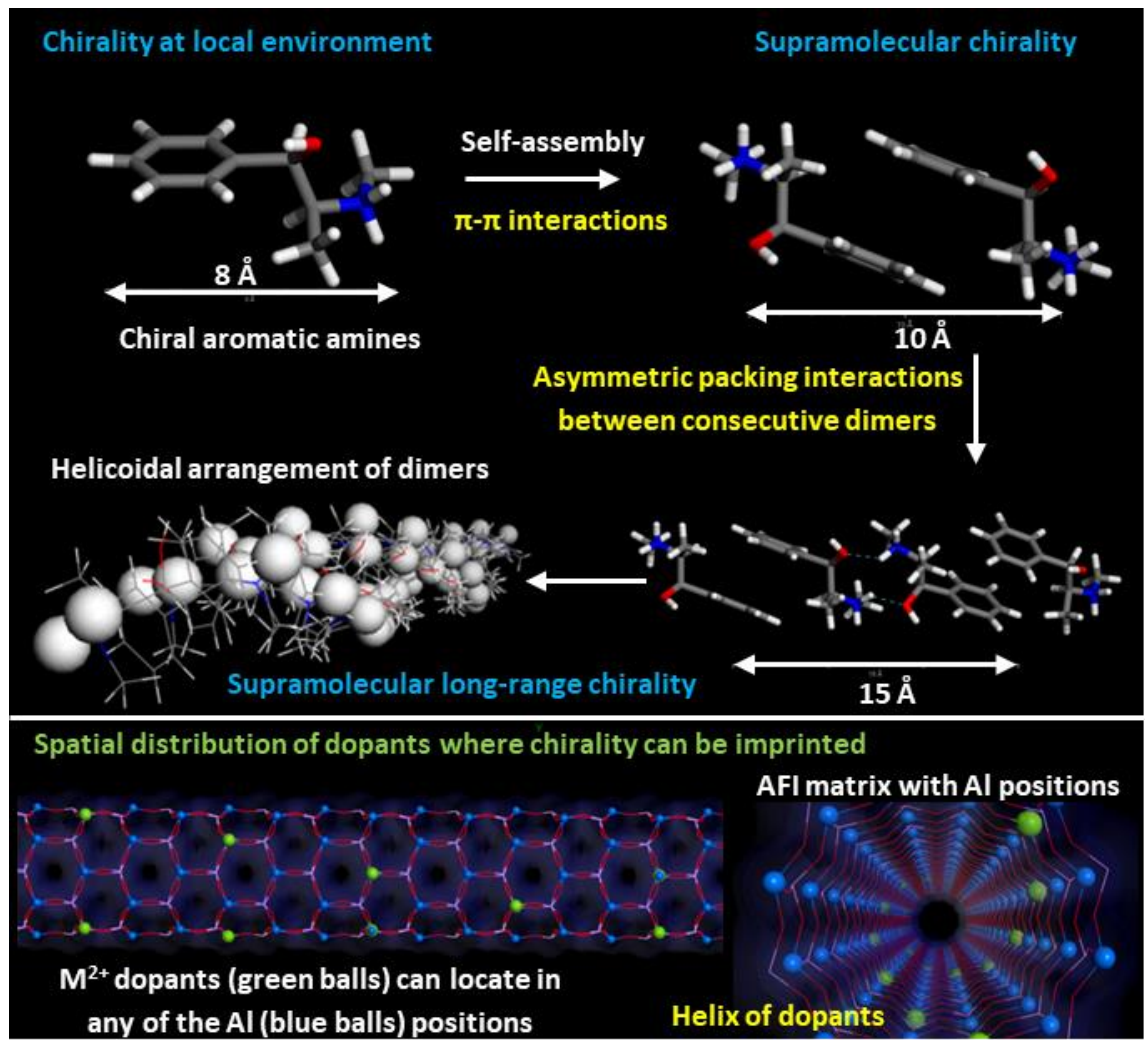

Figure 14. Schematic illustration of the concepts of asymmetric self-assembly of chiral aromatic amines in order to extend the chiral environment into the chiral dimension typical of zeolite structures (top), and AFI matrix with the different positions where dopants can be incorporated (blue balls, Al atoms), and a possible helicoidal distribution of dopants (green balls) (bottom). 


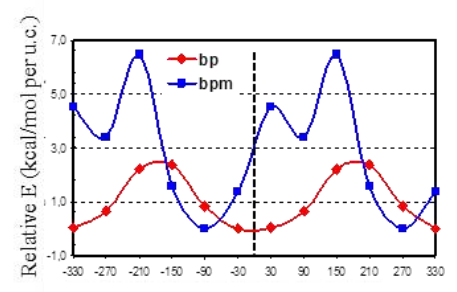

Interdimer angle (deg)

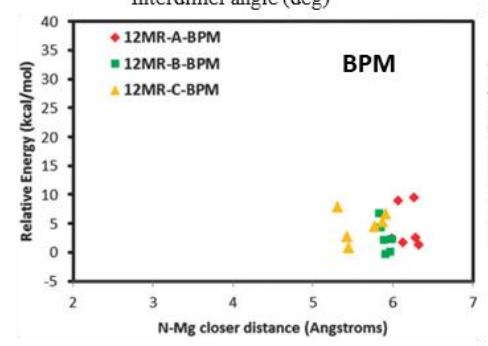

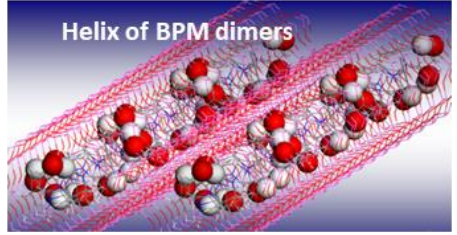

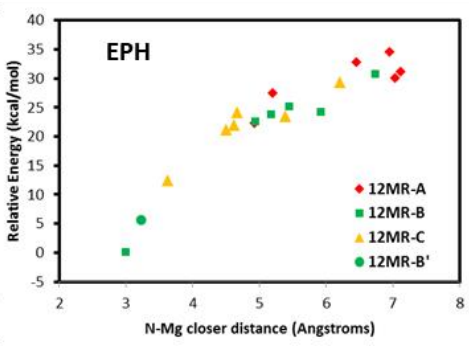

Figure 15. Relative energy diagram for the rotation angle between consecutive dimers for (S)-N-benzylpyrrolidine-2-methanol (BPM, blue) and benzylpyrrolidine (BP, red) (dashed line indicates $0^{\circ}$ rotation) (top-left), and helicoidal arrangement of BPM (top-right). Bottom: stability for different replacement positions of $\mathrm{Al}$ by $\mathrm{Mg}$ as a function of the $\mathrm{Mg} \cdots \mathrm{N}$ interatomic distance for $\mathrm{BPM}$ (left) or ephedrine (EPH, right) (different colors indicate location of $\mathrm{Mg}$ in consecutive 12-rings along the AFI channels). Reproduced (adapted) from [107] and [114] by permission of the PCCP Owner Societies. 


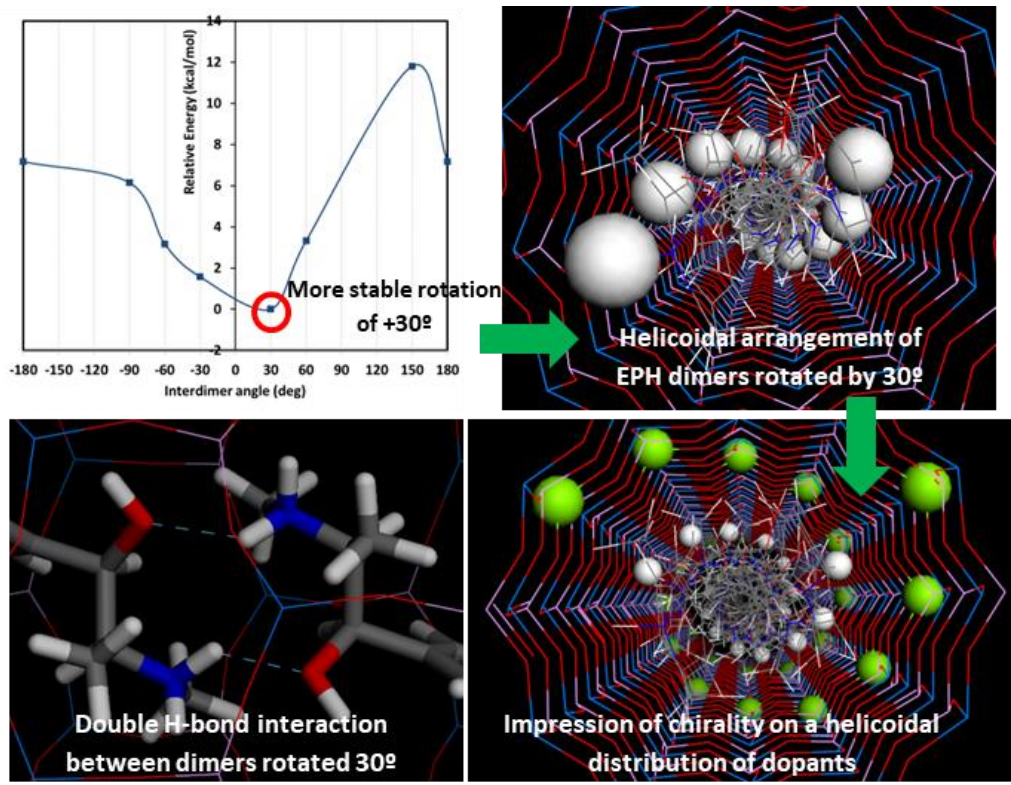

Figure 16. Relative energy diagram for the rotation angle between consecutive dimers for $(1 \mathrm{R}, 2 \mathrm{~S})$-ephedrine (top-left), with the rotation of $30^{\circ}$ being more stable because of a stronger double H-bond interaction between dimers (bottom-left), helicoidal arrangement of EPH dimers rotated by $+30^{\circ}$ (top-right), and impression on a chiral distribution of dopants (bottom-right). Reproduced (adapted) from [114] by permission of the PCCP Owner Societies. 


\section{References}

1. (2002) Chirality in Natural and Applied Science. Editors: Lough WJ, Wainer IW. CRC Press, ISSBN 0-632-05435-2.

2. Chan RS, Ingold CK, Prelog V (1966) Specification of molecular chirality. Angew Chem Int Ed 5(4):385-415.

3. Martin RH (1974) The Helicenes. Angew Chem Int Ed Engl 13(10): 649-660.

4. Gardner M (1964) The Ambidextrous Universe. Symmetry and Asymmetry from Mirror Reflections to Superstrings. 3rd Revised Edition. Penguin Books.

5. Guijarro A, Yus M (2009) The origin of chirality in the molecules of life: a revision from awareness to the current theories and perspectives for this unsolved problem, RSC Publishing, 2009. ISBN: 978-0-85404-156-5.

6. Mason SF (1984) Origins of biomolecular handedness. Nature 311: 19-23.

7. Patil PN (2002) Chirality in Medicinal Chemistry (Chapter 6 in Chirality in Natural and Applied Science). Editors: Lough WJ, Wainer IW. CRC Press, ISSBN 0-63205435-2, pp 139-178.

8. Rouhi M (2005) Top Pharmaceuticals: Thalidomide. Chem Eng News 83:20-6-2005.

9. Thayer AM (2007) Centering On Chirality. Chem Eng News 85:6-8-2007.

10. Haris KDM, Thomas SJM (2009) Selected Thoughts on Chiral Crystals, Chiral Surfaces, and Asymmetric Heterogeneous Catalysis. ChemCatChem 1:223-231.

11. Hazen RM, Sholl DS (2003) Chiral selection on inorganic crystalline surfaces. Nat. Mater. 2:367-374

12. Kavasmaneck PR, Bonner WA (1977) Adsorption of amino acid derivatives by d- and 1-quartz. J Am Chem Soc 99:44-50.

13 Bonner WA (1991) The Origin and Amplification of Biomolecular Chirality. Orig Life Evol Biosph 21:59-111.

14 Hazen RM, Filley TR, Goodfriend GA (2001) Selective Adsorption of L- and Damino acids on calcite: Implication for Biochemical Homochirality. Proc Natl Acad Sci U S A 98(10):5487-5490.

15. Moliner M, Rey F, Corma A (2013) Towards the rational design of efficient organic structure-directing agents for zeolite synthesis. Angew Chem Int Ed 52:13880-13889.

16. Davis ME (2014) Zeolites from a materials chemistry perspective. Chem Mater 26:239-245.

17. Davis ME (2003) Reflections on routes to enantioselective solid catalysts. Top Catal 25:3-7.

18. Yu J, Xu R (2008) Chiral zeolitic materials: Structural insights and synthetic challenges. J Mat Chem 18:4021-4030.

19. Dubbeldam D, Calero S, Vlugt TJH (2014) Exploring new methods and materials for enantioselective separations and catalysis. Mol Simul 40:585-598.

20. Mc Morn P, Hutchings GJ (2004) Heterogeneous enantioselective catalysts: strategies for the immobilisation of homogeneous catalysts. Chem Soc Rev 33:108-122.

21. Davis ME (1998) Zeolite-based catalysts for chemicals synthesis. Microporous Mesoporous Mater. 21:173-182.

22. Treacy MMJ, Newsam JM (1988) 2 New 3-dimensional 12-ring zeolite frameworks of which zeolite beta is a disordered intergrowth. Nature 332:249-251.

23. Rajic N, Logar NZ, Kaucic V (1995) A novel open framework zincophosphate - synthesis and characterization. Zeolites 15:672-678. 
24. Cheetham AK, Fjellvag H, Gier TE et al (2001) Very open microporous materials: from concept to reality. Stud Surf Sci Catal 135:158-158.

25. Bu X, Feng P, Gier TE et al (1998) Hydrothermal synthesis and structural characterization of zeolite-like structures based on gallium and aluminum germinates. J Am Chem Soc 120:13389-13397.

26. Rouse RC, Peacor DR (1986) Crystal structure of the zeolite mineral goosecreekite, $\mathrm{CaAl}_{2} \mathrm{Si}_{6} \mathrm{O}_{16} \cdot 5 \mathrm{H}_{2} \mathrm{O}$. Am Mineral 71:1494-1501.

27. Broach RW, Kirchner RM (2011) Structures of the $\mathrm{K}^{+}$and $\mathrm{NH}_{4}{ }^{+}$forms of Linde $\mathrm{J}$. Microporous Mesoporous Mat. 143:398-400.

28. Song XW, Li Y, Gan L et al (2009) Heteroatom-stabilized chiral framework of aluminophosphate molecular sieves. Angew Chem Int Ed 48:314-317.

29. Sun J, Bonneau C, Cantín A et al (2009) The ITQ-37 mesoporous chiral zeolite. Nature 458:1154-1157.

30. Tang LQ, Shi L, Bonneau C et al (2008) A zeolite family with chiral and achiral structures built from the same building layer. Nat Mater 7:381-385.

31. Rojas A, Camblor MA (2012) A Pure Silica Chiral Polymorph with Helical Pores. Angew Chem Int Ed 51:3854-3856.

32. Rojas A, Arteaga O, Kahr B et al (2013) Synthesis, Structure and optical activity of HPM-1, a pure silica chiral zeolite. J Am Chem Soc 135:11975-11984.

33. Liu X, Xing Y, Wang X et al (2010) Chirality and magnetism of an open-framework cobalt phosphite containing helical channels from achiral materials. Chem Commun 46:2614-2616.

34. Dryzun C, Mastai Y, Shvalb A et al (2009) Chiral silicate zeolites. J Mat Chem 19:2062-2069.

35. Zhang J, Chen SM, Bu XH (2009) Nucleotide-Catalyzed Conversion of Racemic Zeolite-Type Zincophosphate into Enantioenriched Crystals. Angew Chem Int Ed 49:6049-6051

36. Special Issue (2014) Metal-organic frameworks. Chem Soc Rev 43:5403-6176.

37. Ma L, Abney C, Lin W (2009) Enantioselective catalysis with homochiral metalorganic frameworks. Chem Soc Rev 38:1248-1256.

38. Liu Y, Xuan W, Cui Y (2010) Engineering homochiral metal-organic frameworks for heterogeneous asymmetric catalysis and enantioselective separation. Adv Mater 22:4112-4135.

39. Morris RE, Bu XH (2010) Induction of chiral porous solids containing only achiral building blocks. Nat Chem 2:353-361.

40. Lobo RF, Davis ME (1994) Synthesis and characterization of pure-silica and boronsubstituted SSZ-24 using N(16) methylsparteinium bromide as structure-directing agent. Microporous Mater 3:61-69.

41. Yoshikawa M, Wagner P, Lovallo M et al (1998) Synthesis, Characterization, and Structure Solution of CIT-5, a New, High-Silica, Extra-Large-Pore Molecular Sieve. J Phys Chem B 102:7139-7147.

42. Corma A, Díaz-Cabañas MJ, Martínez-Triguero J et al (2002) A large-cavity zeolite with wide pore windows and potential as an oil refining catalyst. Nature 418:514-517.

43. Tsuji K, Wagner P, Davis ME (1999) High-silica molecular sieve syntheses using the sparteine related compounds as structure-directing agents. Microporous Mesoporous Mater 28:461-469.

44. Kubota Y, Helmkamp MM, Zones SI et al (1996) Properties of organic cations that lead to the structure-direction of high-silica molecular sieves. Microporous Mater 6 :213-229. 
45. Lobo RF, Davis ME (1995) CIT-1: A New Molecular Sieve with Intersecting Pores Bounded by 10- and 12-Rings. J Am Chem Soc 117:3766-3769.

46. Xie D, McCusker LB, Baerlocher C et al (2013) SSZ-52, a Zeolite with an 18-Layer Aluminosilicate Framework Structure Related to That of the DeNOx Catalyst Cu-SSZ13. J Am Chem Soc 135:10519-10524.

47. Jiang J, Xu Y, Cheng P et al (2011) Investigation of Extra-Large Pore Zeolite Synthesis by a High-Throughput Approach. Chem Mater 23:4709-4715.

48. García R, Gómez-Hortigüela L, Sánchez F et al (2010) Diastereoselective Structure Directing Effect of (1S,2S)-2-Hydroxymethyl-1-benzyl-1-methylpyrrolidinium in the Synthesis of ZSM-12. Chem Mater 22:2276-2286.

49. García R, Gómez-Hortigüela L, Pérez-Pariente J (2012) Study of the structure directing effect of the chiral cation (1S,2S)-2-hydroxymethyl-1-benzyl-1methylpyrrolidinium in aluminosilicate preparations in the presence of co-structure directing agents. Catal Today 179:16-26.

50. García R, Gómez-Hortigüela L, Sánchez F et al (2011) Structure-direction of chiral 2hydroxymethyl-1-benzyl-1-methylpyrrolidinium in the cotemplated synthesis of ferrierite: Fundaments of diastereo-recognition from non-chiral microporous structures. Microporous Mesoporous Mater 146:57-68.

51. Lobo RF, Tsapatsis M, Freyhardt CC et al (1997) A Model for the Structure of the Large-Pore Zeolite SSZ-31. J Am Chem Soc 119:3732-3744.

52. Brand SK, Schmidt JE, Deem MW et al (2017) Enantiomerically enriched, polycrystalline molecular sieves. Proc Natl Acad Sci U S A in press.

53. Martínez-Franco R, Paris C, Martínez-Triguero J et al (2017) Direct synthesis of the aluminosilicate form of the small pore CDO zeolite with novel OSDAs and the expanded polymorphs. Microporous Mesoporous Mater 246:147-157.

54. Golebiewski WM, Spenser ID (1988) Biosynthesis of the lupine alkaloids. II. Sparteine and lupanine. Can J Chem 66(7):1734-1748.

55. Wagner P, Yoshikawa M, Lovallo M et al (1997) CIT-5: a high-silica zeolite with 14ring pores. Chem Commun 2179-2180.

56. Gómez-Hortigüela L, Álvaro-Muñoz T, unpublished results.

57. Dong $\mathrm{Z}$, Zhao L, Liang $\mathrm{Z}$ et al (2010) $\left[\mathrm{Zn}\left(\mathrm{HPO}_{3}\right)\left(\mathrm{C}_{11} \mathrm{~N}_{2} \mathrm{O}_{2} \mathrm{H}_{12}\right)\right]$ and $\left[\mathrm{Zn}_{3}\left(\mathrm{H}_{2} \mathrm{O}\right)\left(\mathrm{PO}_{4}\right)\left(\mathrm{HPO}_{4}\right)\left(\mathrm{C}_{6} \mathrm{H}_{9} \mathrm{~N}_{3} \mathrm{O}_{2}\right)_{2} \quad\left(\mathrm{C}_{6} \mathrm{H}_{8} \mathrm{~N}_{3} \mathrm{O}_{2}\right)\right]$ : homochiral zinc phosphite/phosphate networks with biofunctional amino acids. Dalton Trans 39:5439-5445.

58. Komura K, Horibe Y, Yajima H et al (2016) Synthesis, crystal structure and characterization of novel open framework CHA-type aluminophosphate involving a chiral diamine. Dalton Trans 45:15193-15202.

59. Gómez-Hortigüela L, Pérez-Pariente J, Blasco T (2007) (S)-(-)-N-benzylpyrrolidine2-methanol: A new and efficient structure directing agent for the synthesis of crystalline microporous aluminophosphates with AFI-type structure. Microporous Mesoporous Mater 100:55-62.

60. Pinar AB, Gómez-Hortigüela L, McCusker LB et al (2011) Synthesis of $\mathrm{Zn}$-containing microporous aluminophosphate with the STA-1 structure. Dalton Trans 40:8125-8131.

61. Álvaro-Muñoz T, López-Arbeloa F, Pérez-Pariente J et al (2014) (1R,2S)-Ephedrine: a new self-assembling chiral template for the synthesis of aluminophosphate frameworks. J Phys Chem C 118:3069-3077.

62. Bernardo-Maestro B, López-Arbeloa F, Pérez-Pariente J et al (2015) Supramolecular chemistry controlled by conformational space during structure-direction of nanoporous 
materials: self-assembly of ephedrine and pseudoephedrine. J Phys Chem C 119:28214-28225.

63. Bernardo-Maestro B, Vos E, López-Arbeloa F et al (2017) Supramolecular Chemistry Controlled by Packing Interactions during Structure-Direction of Nanoporous Materials: Effect of the Addition of Methyl Groups on Ephedrine Derivatives. Microporous Mesoporous Mater 239:432-443.

64. Pinilla-Herrero I, Gómez-Hortigüela L, Márquez-Álvarez C et al (2016) Unexpected crystal growth modifier effect of glucosamine as additive in the synthesis of SAPO-35. Microporous Mesoporous Mater 219:322-326.

65. Nenoff TM, Thoma SG, Provencio P et al (1998) Novel Zinc Phosphate Phases Formed with Chiral d-Glucosamine Molecules. Chem Mater 10:3077-3080.

66. Lin HM, Lii KH (1998) Synthesis and Structure of [(1R,2R)$\left.\mathrm{C}_{6} \mathrm{H}_{10}\left(\mathrm{NH}_{3}\right)_{2}\right]\left[\mathrm{Ga}(\mathrm{OH})\left(\mathrm{HPO}_{4}\right)_{2}\right] \cdot \mathrm{H}_{2} \mathrm{O}$, the first metal phosphate containing a chiral amine. Inorg Chem 37:4220-4222.

67. Abourashed EA, El-Alfy AT, Khan IA et al (2003) Ephedra in Perspective - A Current Review. Phytother Res 17:703-712.

68. Bernardo-Maestro B, Roca-Moreno MD, López-Arbeloa F et al (2016) Supramolecular chemistry of chiral $(1 \mathrm{R}, 2 \mathrm{~S})$-ephedrine confined within the AFI framework as a function of the synthesis conditions. Catal Today 277:9-20.

69 Bernardo-Maestro B, López-Arbeloa F, Pérez-Pariente J et al (2017) Comparison of the Structure-Directing Effect of Ephedrine and Pseudoephedrine during Crystallization of Nanoporous Aluminophosphates. Microporous Mesoporous Mater in press; https://doi.org/10.1016/j.micromeso.2017.04.008.

70. Shi X, Zhu G, Qiu S et al (2004) $\mathrm{Zn}_{2}\left[(\mathrm{~S})-\mathrm{O}_{3} \mathrm{PCH}_{2} \mathrm{NHC}_{4} \mathrm{H}_{7} \mathrm{CO}_{2}\right]_{2}$ : a homochiral 3D zinc phosphonate with helical channels. Angew Chem Int Ed 43:6482-6485.

71. Bernardo-Maestro B, Gómez-Hortigüela L, unpublished results.

72. Morgan K, Gainsford G, Milestone N (1995) A novel layered aluminophosphate $\left[\mathrm{Co}(\mathrm{en})_{3} \mathrm{Al}_{3} \mathrm{P}_{4} \mathrm{O}_{16} \cdot 3 \mathrm{H}_{2} \mathrm{O}\right]$ assembled about a chiral metal complex. J Chem Soc Chem Commun 425-426.

73. Bruce DA, Wilkinson AP, White MG, Bertrand JA (1995) The synthesis and structure of a chiral layered aluminophosphate containing the template Co(tn $)_{3}{ }^{3+}$. J Chem Soc Chem Commun 2059-2060.

74. Bruce DA, Wilkinson AP, White MG et al (1996) The synthesis and characterization of an aluminophosphate with chiral layers; trans-Co(dien $)_{2} \cdot \mathrm{Al}_{3} \mathrm{P}_{4} \mathrm{O}_{16} \cdot 3 \mathrm{H}_{2} \mathrm{O}$. J Solid State Chem 125:228-233.

75. Gray MJ, Jasper JD, Wilkinson AP et al (1997) Synthesis and Synchrotron microcrystal structure of an aluminophosphate with chiral layers containing $\Lambda$ tris(ethylendiamine)cobalt(III). Chem Mater 9:976-980.

76. Yu J, Wang Y, Shi Z et al (2001) Hydrothermal synthesis and characterization of two new zinc phosphates assembled about a chiral metal complex: $\left[\mathrm{Co}^{\mathrm{II}}(\mathrm{en})_{3}\right]_{2}\left[\mathrm{Zn}_{6} \mathrm{P}_{8} \mathrm{O}_{32} \mathrm{H}_{8}\right]$ and $\left[\mathrm{Co}^{\mathrm{III}}(\mathrm{en})_{3}\right]\left[\mathrm{Zn}_{8} \mathrm{P}_{6} \mathrm{O}_{24} \mathrm{Cl}\right] \cdot 2 \mathrm{H}_{2} \mathrm{O}$. Chem Mater 13:2972-2978.

77. Wang Y, Yu J, Li Y et al (2003) Chirality Transfer from Guest Chiral metal complexes to inorganic framework: the role of hydrogen bonding. Chem Eur J 9:5048-5055.

78. Chen P, Li J, Yu J et al (2005) The synthesis and structure of a chiral 1D aluminophosphate chain compound: $d$-Co(en $)_{3}\left[\mathrm{AlP}_{2} \mathrm{O}_{8}\right] \cdot 6.5 \mathrm{H}_{2} \mathrm{O}$. J Solid State Chem 178:19291934.

79. Wang Y, Yu J, Guo M et al (2003) $\left[\mathrm{Zn}_{2}\left(\mathrm{HPO}_{4}\right)_{4}\right]\left[\mathrm{Co}(\text { dien })_{2}\right] \cdot \mathrm{H}_{3} \mathrm{O}$ : a zinc phosphate with multidirectional intersecting helical channels. Angew Chem Int Ed 42:4089-4092. 
80. Han Y, Li Y, Yu J et al (2011) A Gallogermanate Zeolite Constructed Exclusively by Three-Ring Building Units. Angew Chem Int Ed 50:3003-3005.

81. Xu Y, Li Y, Han Y et al (2013) A Gallogermanate Zeolite with Eleven-MemberedRing Channels. Angew Chem Int Ed 52:5501-5503.

82. Balkus KJ, Hargis CD, Kowalak S (1992) Synthesis of NaX Zeolites with Metallophthalocyanines. ACS Symp Ser 499:347-354.

83. Jasper JD, Wilkinson AP (1998) Synthesis of Low-Dimensional Aluminophosphates from Higher Dimensional Precursors: Conversion of $\Lambda, \Delta-\mathrm{Co}(\mathrm{en})_{3}\left[\mathrm{Al}_{3} \mathrm{P}_{4} \mathrm{O}_{16}\right] \cdot \mathrm{xH}_{2} \mathrm{O}$ to the Chain Compound $\Lambda, \Delta-\mathrm{Co}(\mathrm{en})_{3}\left[\mathrm{AlP}_{2} \mathrm{O}_{8}\right] \cdot \mathrm{xH}_{2} \mathrm{O}$. Chem Mater 10:1664-1667.

84. Stalder SM, Wilkinson AP (1997) Synthesis and Characterization of a Chiral 3DFramework Material: $d$-Co(en $)_{3}\left[\mathrm{H}_{3} \mathrm{Ga}_{2} \mathrm{P}_{4} \mathrm{O}_{16}\right]$. Chem Mater 9:2168-2173.

85. Wang Y, Yu J, Li Y et al (2003) Synthesis and characterization of a new layered gallium phosphate $\left[\mathrm{Co}(\mathrm{en})_{3}\right]\left[\mathrm{Ga}_{3}\left(\mathrm{H}_{2} \mathrm{PO}_{4}\right)_{6}\left(\mathrm{HPO}_{4}\right)_{3}\right]$ templated by cobalt complex. J Solid State Chem 170:176-181.

86. Yang G, Sevov SC (2001) [Co(en $\left.)_{3}\right]\left[\mathrm{B}_{2} \mathrm{P}_{3} \mathrm{O}_{11}(\mathrm{OH})_{2}\right]$ : A Novel Borophosphate Templated by a Transition-Metal Complex. Inorg Chem 40:2214-2215.

87. Wadlinger RL, Kerr GT, Rosinski EJ (1967) Catalytic composition of a crystalline zeolite. US patent 3,308,069.

88. Newsam JM, Treacy MMJ, Koetsier WT et al (1988) Structural characterization of zeolite-Beta. Proc R Soc London Ser A 420:375-405.

89. Higgins JB, LaPierre RB, Schlenker JL et al (1988) The framework topology of zeolite-Beta. Zeolites 8:446-452.

90. Conradsson T, Dadachov MS, Zou XD (2000) Synthesis and structure of $\left(\mathrm{Me}_{3} \mathrm{~N}\right)_{6}\left[\mathrm{Ge}_{32} \mathrm{O}_{64}\right]\left(\mathrm{H}_{2} \mathrm{O}\right)_{4.5}$, a thermally stable novel zeotype with 3D interconnected 12ring channels. Microporous Mesoporous Mat. 41:183-191.

91. Davis ME, Lobo RF (1992) Zeolite and molecular sieve synthesis. Chem Mater 4:756-768.

92. Clark LA, Chempath S, Snurr RQ (2005) Simulated adsorption properties and synthesis prospects of homochiral porous solids based on their heterochiral analogs. Langmuir 21:2267-2272.

93. Manning MP, Warzywoda J, Karahan O et al (2004) Enantioselective adsorption of hydrobenzoin on zeolite beta. Stud Surf Sci Catal 154:1957-1960.

94. Takagi Y, Komatsu T, Kitabata Y (2008) Crystallization of zeolite beta in the presence of chiral amine or rhodium complex. Microporous Mesoporous Mater 109:567-576.

95. Camblor MA, Corma A, Valencia S (1996) Spontaneous nucleation and growth of pure silica zeolite-Beta free of connectivity defects. Chem Commun 20:2365-2366.

96. Xia QH, Shen SC, Song J et al (2003) Structure, morphology, and catalytic activity of $\beta$ zeolite synthesized in a fluoride medium for asymmetric hydrogenation. J Catal 219:74-84

97. Taborda F, Willhammar T, Wang Z et al (2011) Synthesis and characterization of pure silica zeolite beta obtained by an aging-drying method. Microporous Mesoporous Mater 143:196-205.

98. Taborda F, Wang Z, Willhammar T et al (2012) Synthesis of Al-Si-beta and Ti-Sibeta by the aging-drying method. Microporous Mesoporous Mater 150:38-46.

99. Tong M, Zhang D, Fan W et al (2015) Synthesis of chiral polymorph A-enriched zeolite Beta with an extremely concentrated fluoride route. Sci Rep 5:11521.

100. Tong M, Zhang D, Zhu L et al (2016) An elaborate structure investigation of the chiral polymorph A-enriched zeolite beta. CrystEngComm 18:1782-1789. 
101. Lu T, Xu R, Yan W (2016) Co-templated synthesis of polymorph A-enriched zeolite beta. Microporous Mesoporous Mater 226:19-24.

102. Qian K, Li J, Jiang J et al (2012) Synthesis and characterization of chiral zeolite ITQ-37 by using achiral organic structure-directing agent. Microporous Mesoporous Mater 164:88-92.

103. Chen FJ, Gao ZH, Liang LL et al (2016) Facile preparation of extra-large pore zeolite ITQ-37 based on supramolecular assemblies as structure-directing agents. CrystEngComm 18:2735-2741.

104. Zhang N, Shi L, Yu T et al (2015) Synthesis and characterization of pure STWzeotype germanosilicate, $\mathrm{Cu}$ - and Co-substituted STW-zeotype materials. J Solid State Chem 225:271-277

105. Schmidt JE, Deem MW, Davis ME (2014) Synthesis of a specified, silica molecular sieve using computationally predicted organic structure-directing agents. Angew Chem Int Ed 53:8372-8374.

106. Castillo JM, Vlugt TJH, Dubbeldam D et al (2010) Performance of Chiral Zeolites for enantiomeric separation revealed by molecular simulation. J Phys Chem C 114:22207-22213.

107. Gómez-Hortigüela L, Corà F, Catlow CRA et al (2006) Computational study of a chiral supramolecular arrangement of organic structure directing molecules for the AFI structure. Phys Chem Chem Phys 8:486-493.

108. Gómez-Hortigüela L, Corà F, Pérez-Pariente J (2012) Chiral distributions of dopants in microporous materials: a new concept of chirality. Microporous Mesoporous Mater $155: 14-15$

109. Comyns AE (2011) Chiral zeolites, a novel approach. Microporous Mesoporous Mater 138:243.

110. Gómez-Hortigüela L, Pinar AB, Corà $\mathrm{F}$ et al (2010) Dopant-siting selectivity in nanoporous catalysts: control of proton accessibility in zeolite catalysts through the rational use of templates. Chem Commun 46:2073-2075.

111. Pinar AB, Gómez-Hortigüela L, McCusker LB et al (2013) Controlling the aluminum distribution in the zeolite ferrierite via the organic structure directing agent. Chem Mater 25:3654-3661.

112. Gómez-Hortigüela L, Pérez-Pariente J, López-Arbeloa F (2009) Aggregation behavior of (S)-(-)-Nbenzylpyrrolidine-2-methanol in the synthesis of the AFI structure in the presence of dopants. Microporous Mesoporous Mater 119:299-305

113. Gómez-Hortigüela L, Lopez Arbeloa F, Márquez-Álvarez C et al (2013) Effect of fluorine and molecular charge-state on the aggregation behavior of (S)-(-)-Nbenzylpyrrolidine-2-methanol confined within the AFI nanoporous structure. J Phys Chem C 117:8832-8839.

114. Gómez-Hortigüela L, Álvaro-Muñoz T, Bernardo-Maestro B et al (2015) Towards chiral distributions of dopants in microporous frameworks: helicoidal supramolecular arrangement of (1R,2S)-ephedrine and transfer of chirality. Phys Chem Chem Phys 17:348-357. 